\title{
Photometric Effects and Voronoi-Diagrams as a Mixed Model for the Spatial Distribution of Galaxies
}

\author{
L. Zaninetti*
}

Dipartimento di Fisica, via P.Giuria 1, I-10125 Turin, Italy

\begin{abstract}
We review the model of the Voronoi Diagrams which allows to reproduce the large-scale structures of our universe as given by the astronomical catalogs. The observed number of galaxies in a given solid angle with a chosen flux/magnitude versus the redshift presents a maximum that is a function of the flux/magnitude ; it can be explained by a detailed analysis of the standard luminosity function for galaxies as well by two new luminosity function for galaxies. The current status of the research on the statistics of the Voronoi Diagrams is reviewed.
\end{abstract}

Keywords: Stochastic processes, Distribution theory and Monte Carlo studies, Patterns, Structures and organization in complex systems, Clusters of galaxies, Cosmology.

\section{INTRODUCTION}

During the last thirty years the spatial distribution of galaxies has been investigated from the point of view of geometrical and physical theories. One first target was to reproduce the two-point correlation function $\xi(r)$ for galaxies which on average scales as $\approx\left(\frac{r}{5.7 M p c}\right)^{-1.8}$, see $[1$, 2]. The statistical theories of spatial galaxy distribution can be classified as

- Levy flights: the random walk with a variable step length can lead to a correlation function in agreement with the observed data, see [3-6].

- Percolation: the theory of primordial explosions can lead to the formation of structures, see [7, 8]. Percolation is also used as a tool to organize : (i) the mass and galaxy distributions obtained in 3D simulations of cold dark matter (CDM) and hot dark matter (HDM), see [9], (ii) the galaxy groups and clusters in volume-limited samples of the Sloan Digital Sky Survey (SDSS), see [10].

- Statistical approach The statistics of the voids between galaxies was analyzed in the Center for Astrophysics Redshift survey, see [11, 12], in the IRAS Point Source Catalog Redshift Survey (PSCz) and Updated Zwicky Catalog of Galaxies (UZC), see [13] and in the Sloan Digital Sky Survey, see [14].

The geometrical models are well represented by the concept of Voronoi Diagrams, after the two historical records by $[15,16]$. The concept of Voronoi Diagrams dates back to the vortex theory applied to the solar system as developed in the 17th century, see Fig. (1) extracted from $[17,18]$.

*Address correspondence to this author at the Dipartimento di Fisica, via P.Giuria 1, I-10125 Turin, Italy; Tel: 0116707460; Fax: 0116707020;

E-mail: zaninetti@ph.unito.it
The first application of the Voronoi Diagrams to the astrophysics is due to [19]. The applications of the Voronoi Diagrams to the galaxies started with [20], where a sequential clustering process was adopted in order to insert the initial seeds, and they continued with [21- 35]. An updated review of the 3D Voronoi Diagrams applied to cosmology can be found in $[33,34]$. The 3D Voronoi tessellation was first applied to identify groups of galaxies in the structure of a super-cluster, see [36-42]. The physical models that produce the observed properties of galaxies are intimately related, for example through the Lagrangian approximation, and can be approximately classified as

- Cosmological N-body: Through N-body experiments by ? it is possible to simulate groups which are analogous to the studies of groups among bright Zwicky-catalog galaxies, $[43,44]$ or covariance functions in simulations of galaxy clustering in an expanding universe which are found to be power laws in the nonlinear regime with slopes centered on 1.9 [45]. Using gigaparticle N-body simulations to study galaxy cluster populations in Hubble volumes, [46] created mock sky surveys of dark matter structure to $\mathrm{z}=1.4$ over $10000^{\circ}$ sq. deg and to $\mathrm{z}=0.5$ over two full spheres. In short, $\mathrm{N}$-body calculations seek to model the full nonlinear system by making discrete the matter distribution and following its evolution in a Lagrangian fashion, while N-body simulations are usually understood to concern gravity only.

- Dynamical Models: Starting from a power law of primordial inhomogeneities it is possible to obtain a twopoint correlation function for galaxies with an exponent similar to that observed, see [47-49].

Another line of work is to assume that the velocity field is of a potential type; this assumption is called the Zel'dovich approximation, see [50-52].The Zel'dovich formalism is a Lagrangian approximation of the fully nonlinear set of equations. In this sense it is "gravity" only and does not include a pressure term. 


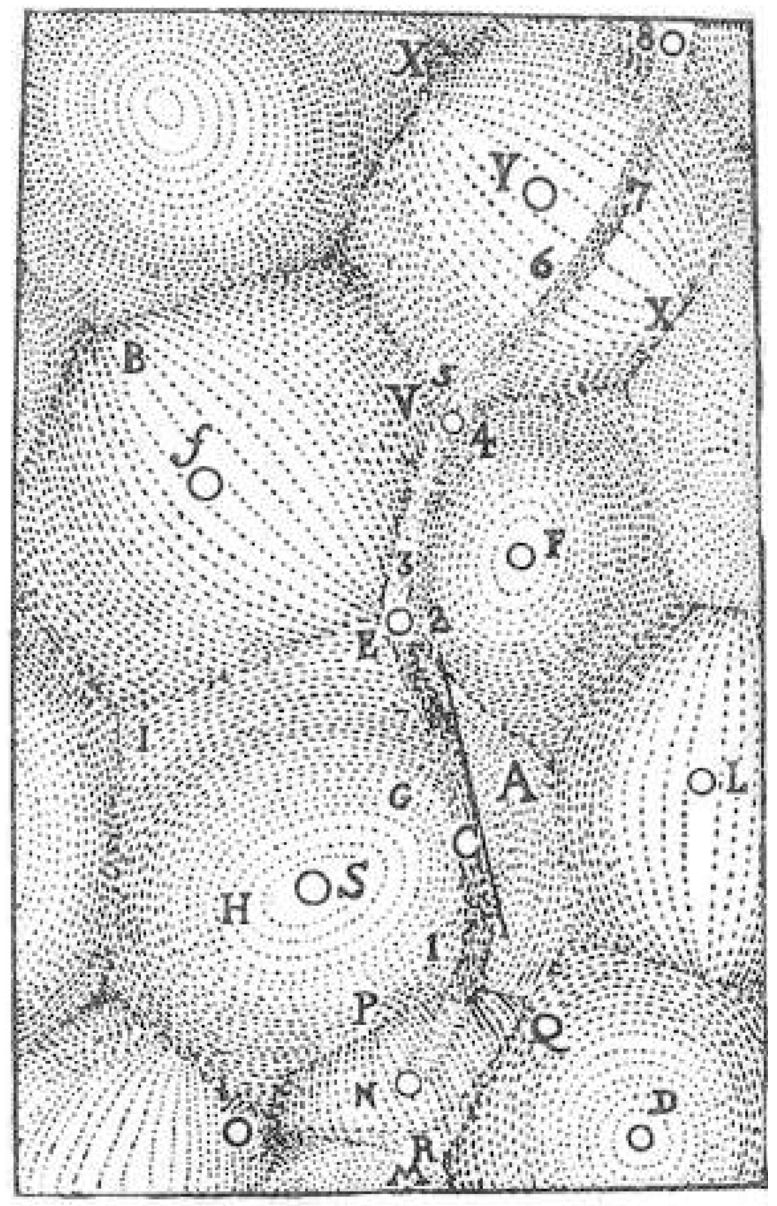

Fig. (1). " God first partitioned the plenum into equal-sized portions, and then placed these bodies into various circular motions that, ultimately, formed the three elements of matter and the vortex systems" ,extracted from [17], volume III , article 46.

- The halo models: The halo model describes nonlinear structures as virialized dark-matter halos of different mass, placing them in space according to the linear large-scale density field which is completely described by the initial power spectrum, see [53-55]. Fig. (19) in [1], for example, reports the exact nonlinear model matter distribution compared with its halo-model representation.

The absence of clear information on the 3D displacement of the physical results as a function of the redshift and the selected magnitude characterize the cosmological N-body, the dynamical and the hydrodynamical models. This absence of detailed information leads to the analysis of the following questions:

- Is it possible to compare the theoretical and observational number of galaxies as a function of the redshift for a fixed flux/magnitude ?

- What is the role of the Malmquist bias when theoretical and observed numbers of galaxies versus the redshift are compared?

- Is it possible to find an algorithm which describes the intersection between a slice that starts from the center of the box and the faces of irregular Poissonian Voronoi Polyhedrons?
- Is it possible to model the intersection between a sphere of a given redshift and the faces of irregular Poissonian Voronoi Polyhedrons?

- Does the developed theory match the observed slices of galaxies as given, for example, by the $2 \mathrm{dF}$ Galaxy Redshift Survey?

- Does the developed algorithm explain the voids appearance in all sky surveys such as the RC3?

- Is it possible to integrate the usual probability density functions (PDFs) which characterize the main parameters of 2D and 3D Poisson Voronoi tessellation (PVT) in order to obtain an analytical expression for the survival function?

- Is it possible to model the normalized areas of $V_{p}(2,3)$ with the known PDFs?

- Can we transform the normalized volumes and areas into equivalent radius distributions?

- Is it possible to derive the probability density function for the radii of 2D sections in the Poissonian Voronoi Tessellation (PVT) and in a well selected case of non Poissonian Voronoi Tessellation (NPVT)?

- Is it possible to evaluate the probability of having a super-void once the averaged void's diameter is fixed?

- Is it possible to compute the correlation function for galaxies by introducing the concept of thick faces of irregular Voronoi polyhedrons?

- Is it possible to find the acoustic oscillations of the correlation function at $\approx 100 M p c$ in simulated slices of the Voronoi diagrams.

In order to answer these questions, Section 2 briefly reviews the elementary formulas adopted and Section 3 reviews the standard luminosity function for galaxies as well two new ones. The adopted catalogs as well the Malmquist bias were presented in Section 4. An accurate test of the number of galaxies as a function of the redshift is performed on the $2 \mathrm{dF}$ Galaxy Redshift Survey (2dFGRS), see Section 5. Section 6 reports the technique which allows us to extract the galaxies belonging to the Voronoi polyhedron. Section 7 reports the apparent distribution in effective radius of the $3 \mathrm{D}$ PVT as well as their associated survival functions, the fit of the normalized area distribution of the sectional PVT with the Kiang function and the exponential distribution. Section 8 reviews the probability of a plane intersecting a given sphere, the stereological approach, and then insert in the fundamental integral of the stereology the cell's radius of the new PDF. Section 9 simulates the redshift dependence of the 2dFGRS as well as the overall Third Reference Catalog of Bright Galaxies (RC3). Section 10 reports the simulation of the correlation function computed on the thick faces of the Voronoi polyhedron.

\section{USEFUL FORMULAS}

This Section reviews the elementary cosmology adopted and the system of magnitudes.

\subsection{Basic Formulas}

Starting from [56] the suggested correlation between expansion velocity and distance is 
$V=H_{0} D=c_{l} z$

where $H_{0}$ is the Hubble constant $H_{0}=100 h \mathrm{~km} \mathrm{~s}^{-1} \mathrm{Mpc}^{-1}$, with $h=1$ when $h$ is not specified, $D$ is the distance in $M p c, c_{l}$ is the light velocity and $z$ is the redshift defined as

$z=\frac{\lambda_{o b s}-\lambda_{e m}}{\lambda_{e m}}$

with $\lambda_{o b s}$ and $\lambda_{e m}$ denoting respectively the wavelengths of the observed and emitted lines as determined from the lab source, the so called Doppler effect . Concerning the value of $H_{0}$ we will adopt a recent value as obtained by the Cepheidcalibrated luminosity of Type Ia supernovae, see [57],

$H_{0}=(62.3 \pm 5) \mathrm{km} \mathrm{s}^{-1} \mathrm{Mpc}^{-1}$

The quantity $c_{l} z$, a velocity, or $z$, a number , characterizes the catalog of galaxies.

We recall that the galaxies have peculiar velocities, making the measured redshifts a combination of cosmological redshift plus a contribution on behalf of the peculiar velocity.

The maximum redshift here considered is $z \approx 0.1$ meaning a maximum velocity of expansion of $\approx 30000$ $\frac{K m}{s}$; up to that value the space is assumed to be Euclidean. We now evaluate the error connected with the use of the simplest cosmological model. For a zero cosmological constant, $\Lambda$, we have the following expression for the luminosity distance $D_{L}$

$D_{L}=\frac{c_{l} z}{H_{0}}\left\{1+\frac{z\left(1-q_{0}\right)}{\sqrt{1+2 q_{0} z}+1+q_{0} z}\right\}$ for $\Lambda=0$

where $q_{0}$ is the deceleration parameter, see [58, 59].

\subsection{Magnitude System}

The absolute magnitude of a galaxy , $M$, is connected to the apparent magnitude $m$ through the relationship

$M=m-5 \log \left(\frac{c_{l} z}{H_{0}}\right)-25$

In an Euclidean ,non-relativistic and homogeneous universe the flux of radiation, $f$, expressed in $\frac{L_{\odot}}{M p c^{2}}$ units, where $L_{\odot}$ represents the luminosity of the sun, is

$f=\frac{L}{4 \pi D^{2}}$

where $D$ represents the distance of the galaxy expressed in $M p c$, and

$D=\frac{c_{l} z}{H_{0}}$

The relationship connecting the absolute magnitude, $M$, of a galaxy to its luminosity is
$\frac{L}{L}=10^{0.4(M}$

where $M_{\odot}$ is the reference magnitude of the sun at the considered bandpass.

The flux expressed in $\frac{L_{\odot}}{M p c^{2}}$ units as a function of the apparent magnitude is

$f=7.957 \times 10^{8} e^{0.921 M_{\odot}-0.921 m} \frac{L_{\odot}}{M p c^{2}}$,

and the inverse relationship is

$m=M_{\odot} m=M_{\odot}-1.0857 \ln \left(0.1256 \times 10^{-8} f\right)$

The equations in this section will be used in the numerical code which allows us to simulate the large scale structures as a function of $z$ and the selected magnitude.

\section{LUMINOSITY FUNCTION FOR GALAXIES}

This Section reviews the standard luminosity function for galaxies, and two new luminosity function for galaxies in the following $L F$.

\subsection{The Schechter Function}

The Schechter function, introduced by [60], provides a useful fit for the $L F$ of galaxies

$\Phi(L) d L=\left(\frac{\Phi^{*}}{L^{*}}\right)\left(\frac{L}{L^{*}}\right)^{\alpha} \exp \left(-\frac{L}{L^{*}}\right) d L$

here $\alpha$ sets the slope for low values of $L, L^{*}$ is the characteristic luminosity and $\Phi^{*}$ is the normalization. The equivalent distribution in absolute magnitude is

$\Phi(M) d M=(0.4 \ln 10) \Phi^{*} 10^{0.4(\alpha+1)\left(M^{*}-M\right)} \exp \left(-10^{0.4\left(M^{*}-M\right)}\right) d M$

where $M^{*}$ is the characteristic magnitude as derived from the data. The scaling with $h$ is $M^{*}-5 \log _{10} h$ and $\Phi^{*} h^{3}\left[M p c^{-3}\right]$. The joint distribution in $z$ and $f$ for galaxies , see formula (1.104) in [61] or formula (1.117) in [62], is

$\frac{d N}{d \Omega d z d f}=4 \pi\left(\frac{c_{l}}{H_{0}}\right)^{5} z^{4} \Phi\left(\frac{z^{2}}{z_{c r i t}^{2}}\right)$

where $d \Omega, d z$ and $d f$ represent the differential of the solid angle, the redshift and the flux respectively. The critical value of $\mathrm{z}, z_{\text {crit }}$, is

$z_{\text {crit }}^{2}=\frac{H_{0}^{2} L^{*}}{4 \pi f c_{l}^{2}}$

The number of galaxies, $N_{S}\left(z, f_{\min }, f_{\max }\right)$ comprised between a minimum value of flux, $f_{\text {min }}$, and maximum value of flux $f_{\max }$, can be computed through the following integral

$N_{S}(z)=\int_{f_{\min }}^{f_{\max }} 4 \pi\left(\frac{c_{l}}{H_{0}}\right)^{5} z^{4} \Phi\left(\frac{z^{2}}{z_{\text {crit }}^{2}}\right) d f$ 
This integral does not have an analytical solution and we must perform a numerical integration.

The number of galaxies in $z$ and $f$ as given by formula (13) has a maximum at $z=z_{\text {pos-max }}$, where

$$
z_{\text {pos }- \text { max }}=z_{\text {crit }} \sqrt{\alpha+2}
$$

which can be re-expressed as

$z_{\text {pos }- \text { max }}=\frac{\sqrt{2+\alpha} \sqrt{10^{0.4 M} \odot^{-0.4 M^{*}}} H_{0}}{2 \sqrt{\pi} \sqrt{f} c_{l}}$

\subsection{The Mass-Luminosity Relationship}

A new $L F$ for galaxies as derived in [63] is

$\Psi(L) d L=\left(\frac{1}{a \Gamma(c)}\right)\left(\frac{\Psi^{*}}{L^{*}}\right)\left(\frac{L}{L^{*}}\right)^{\frac{c-a}{a}} \exp \left(-\left(\frac{L}{L^{*}}\right)^{\frac{1}{a}}\right) d L$

where $\Psi^{*}$ is a normalization factor which defines the overall density of galaxies, a number per cubic $M p c, 1 / a$ is an exponent which connects the mass to the luminosity and $c$ is connected with the dimensionality of the fragmentation, $c=2 d$, where $d$ represents the dimensionality of the considered space: $1,2,3$. The scaling with $h$ is $M^{*}-5 \log _{10} h$ and $\Psi^{*} h^{3}\left[\mathrm{Mpc}^{-3}\right]$. The distribution in absolute magnitude is

$\Psi(M) d M=$

$\left(0.4 \ln 10 \frac{1}{a \Gamma(c)}\right) \Psi^{*} 10^{0.4\left(\frac{c}{a}\right)\left(M^{*}-M\right)} \exp \left(-10^{0.4\left(M^{*}-M\right)\left(\frac{1}{a}\right)}\right) d M$

This function contains the parameters $M^{*}, a, c$ and $\Psi^{*}$ which are derived from the operation of fitting the observational data.

The joint distribution in $z$ and $f$, in presence of the $\mathcal{M}-L \quad L F$ (equation (18)) is

$\frac{d N}{d \Omega d z d f}=4 \pi\left(\frac{c_{l}}{H_{0}}\right)^{5} z^{4} \Psi\left(\frac{z^{2}}{z_{\text {crit }}^{2}}\right)$

The number of galaxies, $N_{\mathcal{M}-L}\left(z, f_{\min }, f_{\max }\right)$ comprised between $f_{\min }$ and $f_{\max }$, can be computed through the following integral

$$
N_{\odot-L}(z)=\int_{f_{\min }}^{f_{\text {max }}} 4 \pi\left(\frac{c_{l}}{H_{0}}\right)^{5} z^{4} \Psi\left(\frac{z^{2}}{z_{\text {crit }}^{2}}\right) d f
$$

and also in this case a numerical integration must be performed.

The number of galaxies as given by formula (20) has a maximum at $z_{\text {pos-max }}$ where

$$
z_{\text {pos }-\max }=z_{\text {crit }}(c+a)^{a / 2}
$$

which can be re-expressed as

$$
z_{p o s-\max }=\frac{(a+c)^{1 / 2 a} \sqrt{10^{0.4 M} \odot^{-0.4 M^{*}}} H_{0}}{2 \sqrt{\pi} \sqrt{f} c_{l}}
$$

\subsection{The Generalized Gamma Distribution with Four Parameters}

The starting point is the probability density function (in the following PDF) named generalized gamma that we report exactly as in [64]:

$G(x ; a, b, c, k)=\frac{k\left(\frac{x-a}{b}\right)^{c k-1} \mathrm{e}^{-\left(\frac{x-a}{b}\right)^{k}}}{b \Gamma(c)}$

where $\Gamma$ is the gamma function, $a$ is the location parameter, $b$ is the scale parameter, $c$ and $k$ are two shape parameters. A $L F$ can be derived inserting $a=0, x=L$ and $b=L^{*}$ :

$\Psi\left(L ; L^{*}, c, k, \Psi^{*}\right)=\Psi^{*} \frac{k\left(\frac{L}{L^{*}}\right)^{c k-1} \mathrm{e}^{-\left(\frac{L}{L^{*}}\right)^{k}}}{L^{*} \Gamma(c)}$

The mathematical range of existence is $0 \leq L<\infty$ and the number of parameters is four because $a=0$ and $\Psi^{*}$ have been added. The averaged luminosity is

$\langle L\rangle=\frac{L^{*} \Gamma\left(\frac{1+c k}{k}\right)}{\Gamma(c)}$

and the mode is at

$L=\left(\frac{c k-1}{k}\right)^{\frac{1}{k}} L^{*}$

The magnitude version of the $L F$ is

$\Psi(M) d M=\frac{\Psi^{*} 0.4 \ln (10) k 10^{-0.4 c k\left(M-M^{*}\right)} \mathrm{e}^{-10^{-0.4\left(M-M^{*}\right) k}}}{\Gamma(c)} d M$

The mode when expressed in magnitude is at

$M=-\frac{1.0857 \ln \left(\frac{c k-1}{k}\right)}{k}+M^{*}$

This function contains the four parameters $c, k, M^{*}$ and $\Psi^{*}$, more details as well other two new $L F$ are reported in [65].

The joint distribution in $z$ and $f$ of the generalized gamma $L F$ is

$\frac{d N}{d \Omega d z d f}=4 \pi\left(\frac{c_{l}}{H_{0}}\right)^{5} z^{4} \Psi\left(\frac{z^{2}}{z_{c r i t}^{2}}\right)$

The number of galaxies, $N_{L F 4}\left(z, f_{\min }, f_{\max }\right)$ of the generalized gamma $L F$ comprised between $f_{\min }$ and $f_{\max }$, can be computed through the following integral:

$$
N_{L F 4}(z)=\int_{f_{\min }}^{f_{\max }} 4 \pi\left(\frac{c_{l}}{H_{0}}\right)^{5} z^{4} \Psi\left(\frac{z^{2}}{z_{\text {crit }}^{2}}\right) d f
$$

and in this case a numerical integration must be performed.

The number of galaxies of the generalized gamma LF as given by formula (30) has a maximum at $z_{\text {pos-max }}$ where 
$z_{\text {pos-max }}=e^{1 / 2 \frac{\ln (1+c k)-\ln (k)}{k}} z_{\text {crit }}$

which can be re-expressed as

$z_{p o s-\max }=\frac{e^{1 / 2 \frac{\ln (1+c k)-\ln (k)}{k}} \sqrt{10^{0.4 M_{\odot}-0.4 M^{*}}} H_{0} k}{2 \sqrt{\pi} \sqrt{f} c_{l}}$

\section{THE ADOPTED CATALOGS}

We now introduce the processed catalogs of galaxies, the statistics of 1024 observed cosmic voids as well the Malmquist bias.

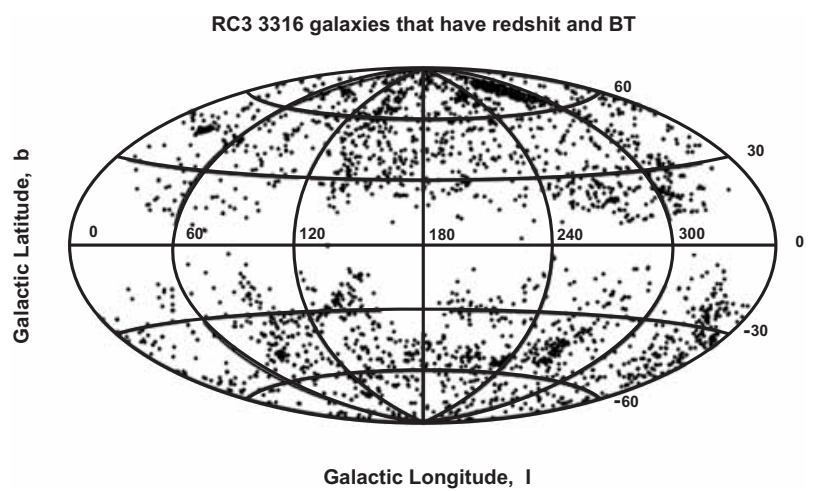

Fig. (2). Hammer-Aitoff projection in galactic coordinates of 3316 galaxies in the RC3 which have BT and redshift. The ZOA is due to our own galaxy.

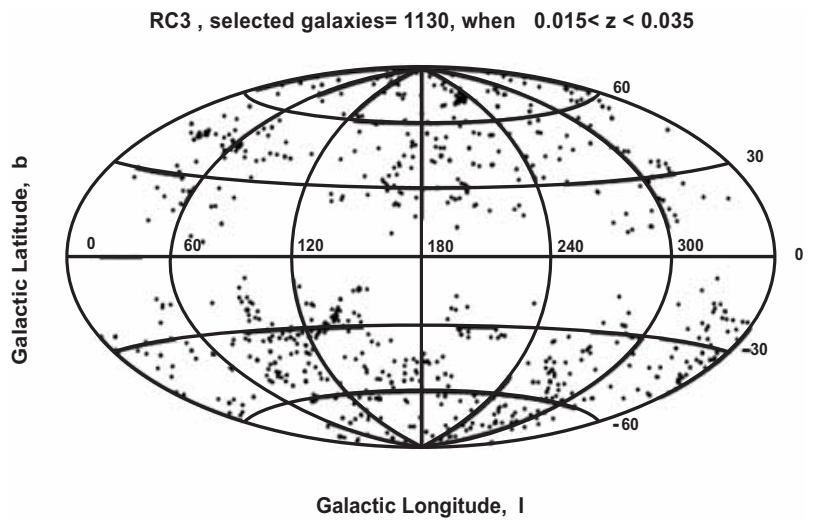

Fig. (3). Hammer-Aitoff projection in galactic coordinates (observational counterpart of $V_{s}(2,3)$ ) of 1130 galaxies in the RC3 which have BT and $0.015<z<0.035$.

\subsection{The Astronomical Catalogs}

A first example is the $2 \mathrm{dFGRS}$ data release available on the web site: http://msowww.anu.edu.au/2dFGRS/. In particular we added together the file parent.ngp.txt which contains 145652 entries for NGP strip sources and the file parent.sgp.txt which contains 204490 entries for SGP strip sources. Once the heliocentric redshift was selected we processed 219107 galaxies with $0.001 \leq z \leq 0.3$.

A second example is the catalog RC3, see [66], which is available at the following address http://vizier.ustrasbg.fr/viz-bin/VizieR?-source=VII/155.
This catalog attempts to be reasonably complete for galaxies having apparent diameters larger than 1 arcmin at the D25 isophotal level and total B-band magnitudes BT, brighter than about 15.5, with a redshift not in excess of $15000 \mathrm{~km} / \mathrm{s}$. All the galaxies in the RC3 catalog which have redshift and BT are reported in Fig. (2). In the case of RC3 the covered area is $4 \pi$ steradians with the exclusion of the Zone of Avoidance (ZOA).

Fig. (3) reports the RC3 galaxies in a given window in $z$. A third example is the Two-Micron All Sky Survey (2MASS) which has instruments in the infrared (1-2.2 $\mu \mathrm{m}$ ) and therefore detects the galaxies in the so called "Zone of Avoidance" , see [67, 68]. Fig. (4) reports a spherical cut at a given radius of the Local Super-cluster (LSC) according to 2MASS Redshift Survey (2MRS), which is available online at https://www.cfa.harvard.edu/ huchra. In the case of 2MRS the covered area is $4 \pi$ steradians.

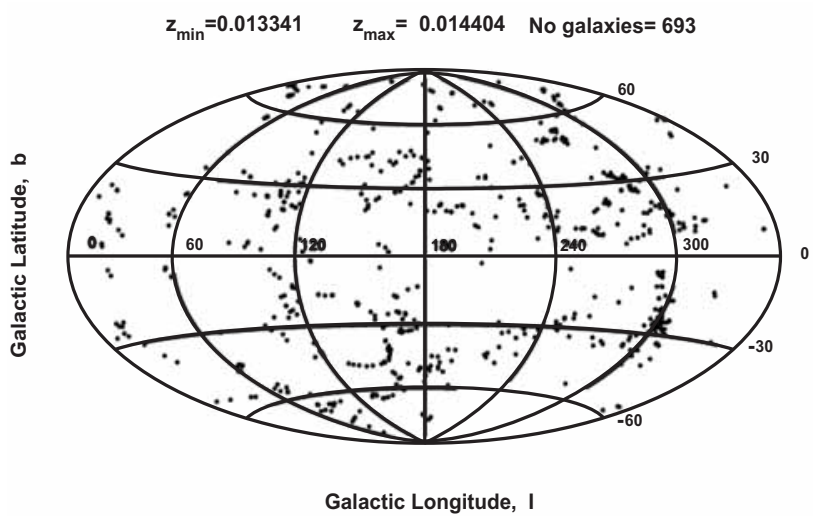

Fig. (4). Hammer-Aitoff projection in galactic coordinates of a spherical cut of the Large Super Cluster data (LSC) at $0.0133 \leq z \leq 0.0144$ or $56.9 M p c \leq D \leq 61.67$.

A fourth example is the second CFA2 redshift Survey, which started in 1984, and produced slices showing that the spatial distribution of galaxies is not random but distributed on filaments that represent the 2D projection of 3D bubbles. We recall that a slice comprises all the galaxies with magnitude $m_{b} \leq 16.5$ in a strip of $6^{\circ}$ wide and about $130^{\circ}$ long. One of such slice (the so called first CFA strip) is visible at the following address http://cfa-www.harvard.edu/ huchra/zcat/ ; more details can be found in [69]. The already mentioned slice can be down-loaded from http://cfawww.harvard.edu/ huchra/zcat/n30.dat/ .

\subsection{Statistics of the Voids}

The distribution of the effective radius and the radius of the maximal enclosed sphere between galaxies of the Sloan Digital Sky Survey Data Release 7 (SDSS DR7) has been reported in [14]. This catalog contains 1054 voids: Table 1 shows the basic statistical parameters of the effective radius.

\subsection{Malmquist Bias}

This bias was originally applied to the stars, [70, 71], and was then applied to the galaxies by [72]. We now introduce the concept of limiting apparent magnitude and the corresponding completeness in absolute magnitude of the considered catalog as a function of redshift. The observable absolute magnitude as a function of the limiting apparent magnitude, $m_{L}$, is 


$$
M_{L}=m_{L}-5 \log _{10}\left(\frac{c z}{H_{0}}\right)-25
$$

The previous formula predicts, from a theoretical point of view, an upper limit on the absolute maximum magnitude that can be observed in a catalog of galaxies characterized by a given limiting magnitude and Fig. (5) reports such a curve and the galaxies of the 2dFGRS.

Table 1. The Statistical Parameters of the Effective Radius in SDSS DR7

\begin{tabular}{|c|c|}
\hline Parameter & Value \\
\hline Elements & $\mathbf{1 0 2 4}$ \\
\hline \hline Mean & $18.23 \mathrm{~h}^{-1} M p c$ \\
\hline Variance & $23.32 \mathrm{~h}^{-2} M p c^{2}$ \\
\hline Standard deviation & $4.82 \mathrm{~h}^{-1} M p c$ \\
\hline Skewness & 0.51 \\
\hline Kurtosis & 0.038 \\
\hline Maximum value & $34.12 \mathrm{~h}^{-1} M p c$ \\
\hline Minimum value & $9.9 \mathrm{~h}^{-1} M p c$ \\
\hline
\end{tabular}

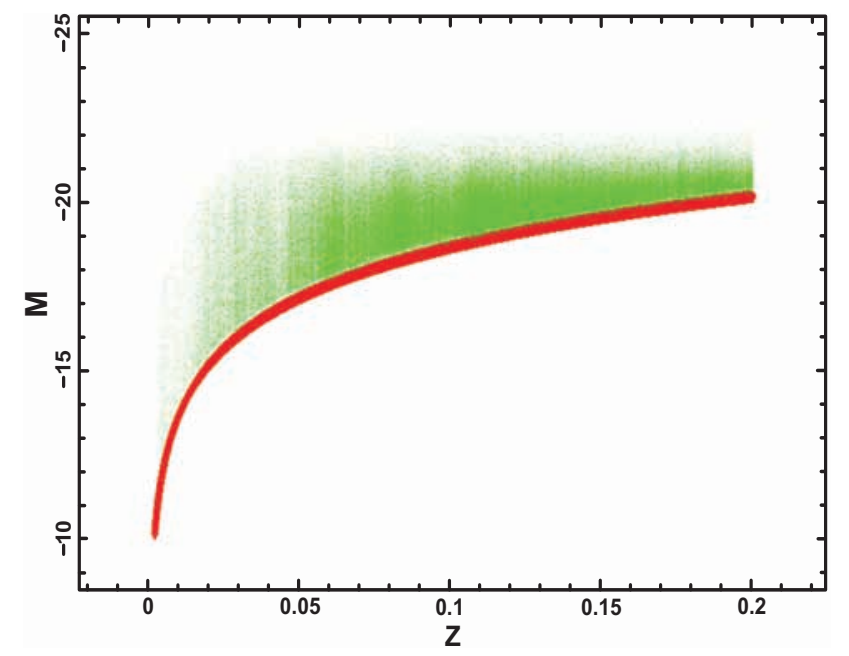

Fig. (5). The absolute magnitude $M$ of 202,923 galaxies belonging to the 2dFGRS when $\mathcal{M}_{\odot}=5.33$ and $H_{0}=66.04 \mathrm{~km} \mathrm{~s}^{-1} \mathrm{Mpc}^{-1}$ (green points). The upper theoretical curve as represented by equation (34) is reported as the red thick line when $m_{L}=19.61$.

The interval covered by the LF of galaxies, $\Delta M$, is defined by

$$
\Delta M=M_{\max }-M_{\min }
$$

where $M_{\max }$ and $M_{\min }$ are the maximum and minimum absolute magnitude of the $L F$ for the considered catalog. The real observable interval in absolute magnitude, $\Delta M_{L}$, is

$$
\Delta M_{L}=M_{L}-M_{\min }
$$

We can therefore introduce the range of observable absolute maximum magnitude expressed in percent, $\varepsilon(z)$, as
$\varepsilon_{s}(z)=\frac{\Delta M_{L}}{\Delta M} \times 100 \%$.

This is a number that represents the completeness of the sample and, given the fact that the limiting magnitude of the 2dFGRS is $m_{L}=19.61$, it is possible to conclude that the 2dFGRS is complete for $z \leq 0.0442$. In the case of the 2MRS the limiting magnitude is $m_{L}=11.19$, and therefore the 2MRS is complete for $z \leq 0.00016$. This efficiency, expressed as a percentage, can be considered to be a version of the Malmquist bias.

Table 2. The Parameters of the Schechter Function for the 2dFGRS as in Madgwick et al. 2002

\begin{tabular}{|c|c|}
\hline Parameter & 2dFGRS \\
\hline \hline $\mathrm{M}^{*}-5 \log _{10} \mathrm{~h}[\mathrm{mags}]$ & $(-19.79 \pm 0.04)$ \\
\hline$\alpha$ & $-1.19 \pm 0.01$ \\
\hline$\Phi^{*}\left[\mathrm{~h}^{3} \mathrm{Mpc}^{-3}\right]$ & $\left((1.59 \pm 0.1) 10^{-2}\right)$ \\
\hline
\end{tabular}

Table 3. The Parameters of the $\mathcal{M}-L \quad L F$ Based on the 2dFGRS Data ( Triplets Generated by the Author)

\begin{tabular}{|c|c|}
\hline & 2dFGRS \\
\hline \hline $\mathrm{c}$ & 0.1 \\
\hline $\mathrm{M}^{*}-5 \log _{10} \mathrm{~h}[\mathrm{mags}]$ & $-19 \pm 0.1$ \\
\hline$\Psi *\left[\mathrm{~h}^{3} \mathrm{Mpc}^{-3}\right]$ & $0.4 \pm 0.01$ \\
\hline $\mathrm{a}$ & $1.3 \pm 0.1$ \\
\hline
\end{tabular}

\section{PHOTOMETRIC MAXIMUM}

The parameters of the Schechter $L F$ concerning the 2dFGRS are reported in Table 2 and those of the $\mathcal{M}-L \quad L F$ are reported in Table $\mathbf{3}$.

It is interesting to point out that other values for $h$ different from 1 shift all absolute magnitudes by $5 \log _{10} h$ and change the number densities by the factor $h^{3}$. Fig. (6) reports the number of observed galaxies of the 2dFGRS catalog for a given apparent magnitude and two theoretical curves as represented by formula (13) which is based on the Schechter $L F$ and formula (20) which is based on the $\mathcal{M}-L L F$. A similar plot can be done for the generalized gamma $L F$, see Fig. (7). The $x^{2}$ analysis allows to conclude that in the two cases here examined the application of the $M-L, L F$ and the generalized gamma LF produce the same or better results in respect to the use of the Schechter LF. More details can be found in $[73,65]$.

The non-homogeneous structure of the universe can be clarified by counting the number of galaxies in one of the two slices of $2 \mathrm{dFGRS}$ as a function of the redshift when a sector with a central angle of $1^{\circ}$ is considered, see Fig. (8).

Conversely, when the two slices are considered together the behavior of the number of galaxies as a function of the redshift is more continuous, see Fig. (9).

\section{THE 3D VORONOI DIAGRAMS}

The faces of the Voronoi Polyhedra share the same property, i.e. they are equally distant from two nuclei. The 
intersection between a plane and the faces produces diagrams which are similar to the edges' displacement in 2D Voronoi diagrams. From the point of view of the observations it is very useful to study the intersection between a slice which crosses the center of the box and the faces of irregular polyhedrons where presumably the galaxies reside. The general definition of the 3D Voronoi Diagrams is given in Section 6.1. The intersection between a slice of a given opening angle, for example $3^{\circ}$, and the faces of the Voronoi Polyhedra can be realized through an approximate algorithm, see next Section 6.2.

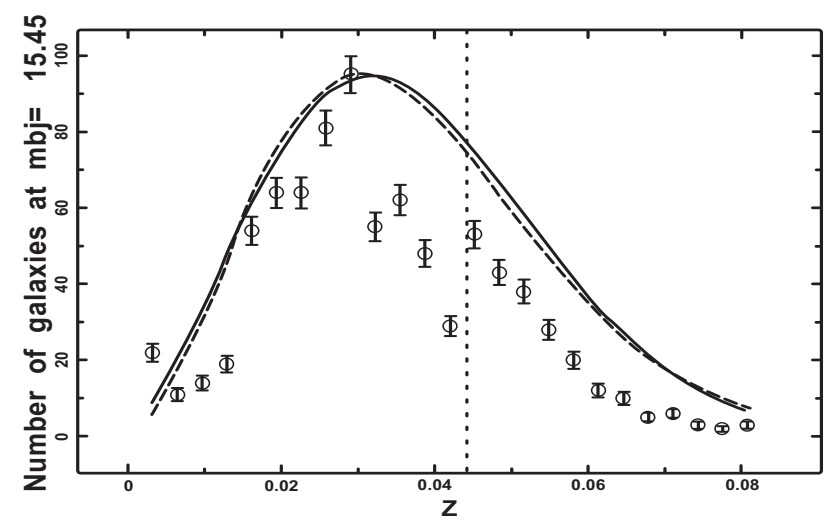

Fig. (6). The galaxies of the $2 \mathrm{dFGRS}$ with $15.27 \leq$ bJmag $\leq 15.65$ or $59253 \frac{L_{\odot}}{M p c^{2}} \leq f \leq 83868 \frac{L_{\odot}}{M p c^{2}}$ (with bJmag representing the relative magnitude used in object selection), are isolated in order to represent a chosen value of $m$ and then organized in frequencies versus heliocentric redshift, (empty circles); the error bar is given by the square root of the frequency. The maximum in the frequencies of observed galaxies is at $\mathrm{z}=0.03$. The theoretical curve generated by the Schechter $L F$ (formula (13) and parameters as in column 2dFGRS of Table 2) is drawn (full line). The theoretical curve generated by the $M-L L F$ (formula (20) and parameters as in column $2 \mathrm{dFGRS}$ of Table 3 ) is drawn (dashed line); $x^{2}=550$ for the Schechter function and $x^{2}=503$ for the $M-L$ function. In this plot $M_{\odot}=5.33$ and $h=0.623$. The vertical dotted line represents the boundary between complete and incomplete samples.

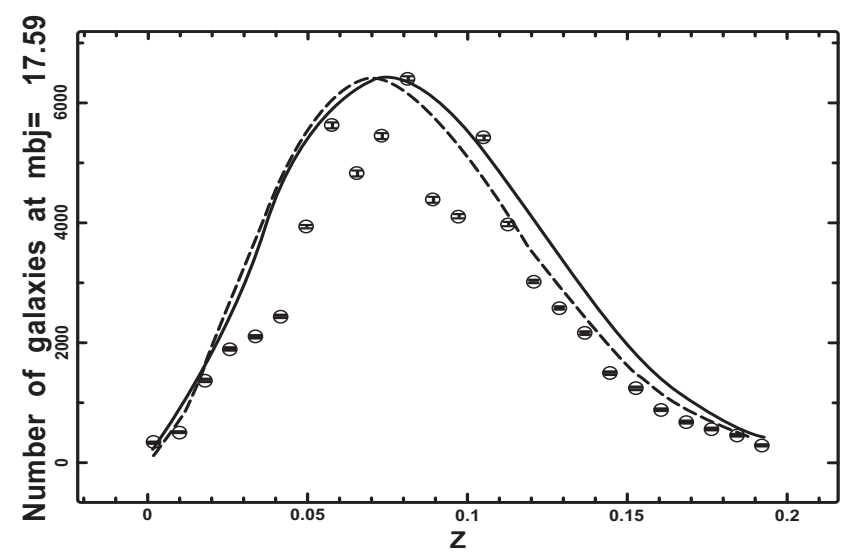

Fig. (7). The galaxies of the 2dFGRS database with $16.77 \leq$ bJmag $\leq 18.40 \quad$ or $\quad 4677 \frac{L_{\odot}}{M p c^{2}} \leq f \leq 21087 \frac{L_{\odot}}{M p c^{2}} \quad$ (with bJmag representing the relative magnitude used in object selection), are isolated in order to represent a chosen value of $m$ and then organized as frequency versus heliocentric redshift, (empty circles); the error bar is given by the square root of the frequency. The maximum in the frequencies of observed galaxies is at $\mathrm{z}=0.085$ when $M_{\odot}=5.33$ and $h=1$. The theoretical curve generated by the Schechter function of luminosity (formula (13) and parameters as in column 2dFGRS of Table 2) is drawn (full line). The theoretical curve generated by generalized gamma LF, formula (20), and parameters as in column 2dFGRS of Table 3) is drawn (dashed line); $x^{2}=8078$ for the Schechter function and $\chi^{2}=6654$ for generalized gamma LF.

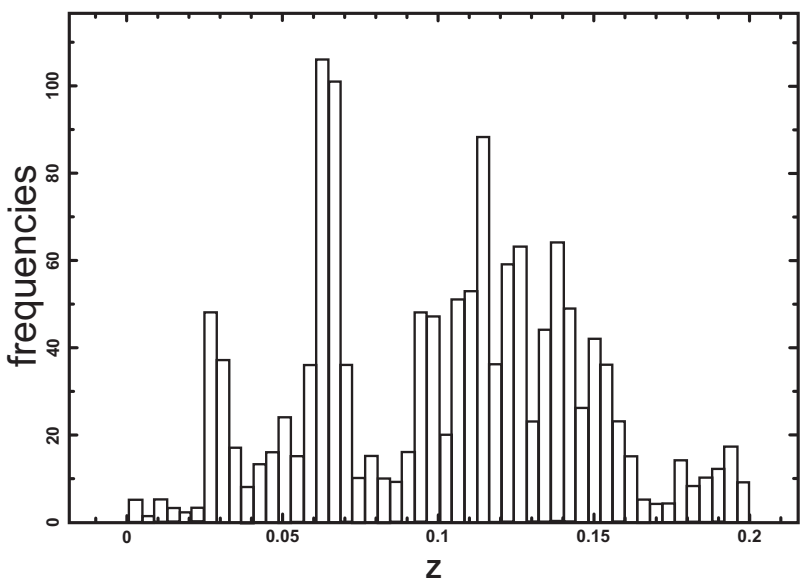

Fig. (8). Histogram (step-diagram) of the number of galaxies of 2dFGRS as a function of the redshift in the slice to the right of Fig. (19), the number of bins is 50 . The circular sector has a central angle of $1^{\circ}$.

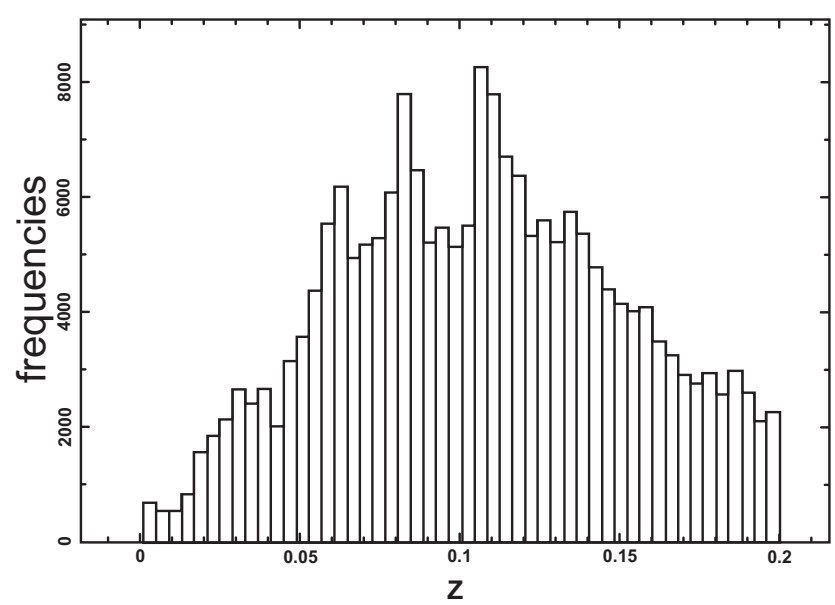

Fig. (9). Histogram (step-diagram) of the number of galaxies of 2dFGRS as a function of the redshift when the two slices of Fig. 19 are added together, the number of bins is 50 .

\subsection{General Definition}

The Voronoi diagram for a set of seeds, $S$, located at position $x_{i}$ in $R^{3}$ space is the partitioning of that space into regions such that all locations within any one region are closer to the generating point than to any other. In the following we will work on a three dimensional lattice defined by pixels $\times$ pixels $\times$ pixels points, $L_{k m n}$. The Voronoi polyhedron $V_{i}$ around a given center $i$, is the set of lattice points $L_{k m n}$ closer to $i$ than to any $j$ : more formally, 
$L_{k m n} \varepsilon V_{i} \leftrightarrow\left|x_{k m n}-x_{i}\right| \leq \mid x_{k m n}-x_{j}$

where $x_{k m n}$ denotes the lattice point position. Thus, the Polyhedra are intersections of half-spaces. Given a center $i$ and its neighbor $j$, the line $i j$ is cut perpendicularly at its midpoint $y_{i j}$ by the plane $h_{i j}$. $H_{i j}$ is the half-space generated by the plane $h_{i j}$, which consists of the subset of lattice points on the same side of $h_{i j}$ as $i$; therefore

$V_{i}=\cap_{j} H_{i j}$

$V_{i}$ is bounded by faces, with each face $f_{i j}$ belonging to a distinct plane $h_{i j}$. Each face will be characterized by its vertexes and edges.

\subsection{The Adopted Algorithm}

Our method considers a 3D lattice with pixels ${ }^{3}$ points: present in this lattice are $N_{s}$ seeds generated according to a random process. All the computations are usually performed on this mathematical lattice; the conversion to the physical lattice is obtained by multiplying the unit by $\delta=\frac{\text { side }}{\text { pixels }-1}$,

where side is the length of the cube expressed in the physical unit adopted. In order to minimize boundary effects introduced by those polyhedron which cross the cubic boundary, the cube in which the seeds are inserted is amplified by a factor amplify. Therefore the $N_{s}$ seeds are inserted in a volume pixels ${ }^{3} \times$ amplify, which is bigger than the box over which the scanning is performed; amplify is generally taken to be equal to 1.2 . This procedure inserts periodic boundary conditions to our cube. A sensible and solid discussion of what such an extension of a cube should be can be found in [74]. The set $S$ of the seeds can be of Poissonian or non-Poissonian type. Adopting the point of view that the universe should be the same from each point of view of the observer the Poissonian seeds can represent the best choice in order to reproduce the large scale structures.

The Poissonian seeds are generated independently on the $X, Y$ and $Z$ axis in 3D through a subroutine which returns a pseudo-random real number taken from a uniform distribution between 0 and 1 . For practical purposes, the subroutine RAN2 was used, see [75]. Particular attention should be paid to the average observed diameter of voids, $D V^{\text {obs }}$, here chosen as

$\overline{D V^{o b s}} \approx 36.46 \mathrm{Mpc} / \mathrm{h}$

see Section 4.2. The number of Poissonian seeds is chosen in such a way that the averaged volume occupied by a Voronoi polyhedron is equal to the averaged observed volume of the voids in the spatial distribution of galaxies; more details can be found in [35].

We now work on a 3D lattice $\mathrm{L}_{k, m, n}$ of pixels ${ }^{3}$ elements. Given a section of the cube (characterized, for example, by $k=\frac{\text { pixels }}{2}$ ) the various $V_{i}$ (the volume belonging to the seed i) may or may not cross the pixels belonging to the two dimensional lattice. A typical example of a 2D cut organized in two strips about $75^{\circ}$ long is visible in Fig. (10) where the Cartesian coordinates $X$ and $Y$ with the origin of the axis at the center of the box has been used. The previous cut has an extension on the $Z$-axis equal to zero.

Conversely Fig. (11) reports two slices of $75^{\circ}$ long and $3^{\circ}$ wide. In this case the extension of the enclosed region belonging to the $Z$-axis increases with distance according to

$\Delta Z=\sqrt{X^{2}+y^{2}} \tan \frac{\alpha}{2}$

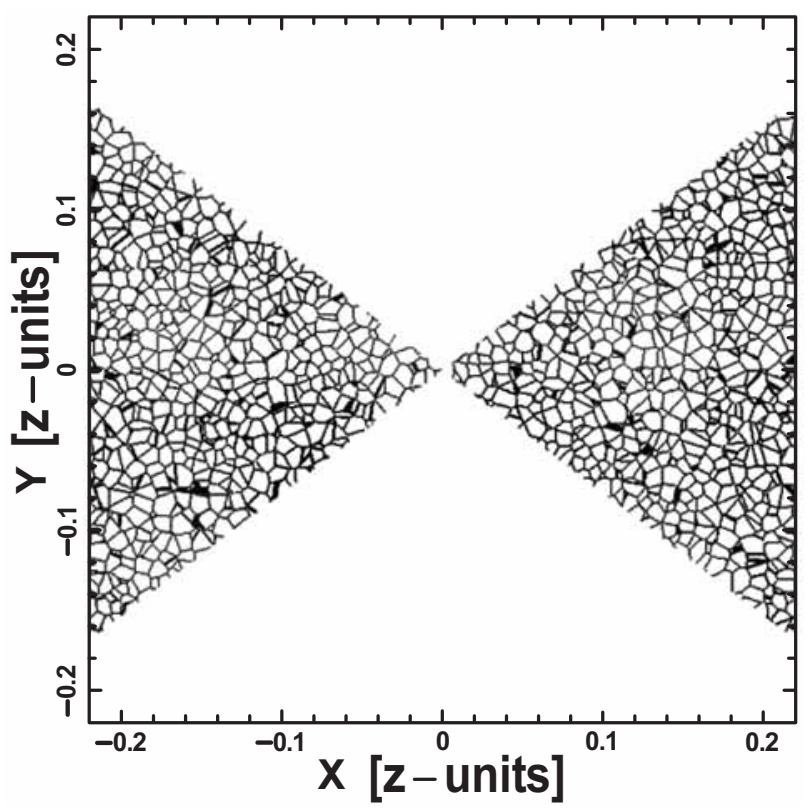

Fig. (10). Portion of the Poissonian Voronoi--diagram $V_{p}(2,3)$; cut on the X-Y plane when two strips of $75^{\circ}$ are considered. The parameters are pixels $=600, N_{s}=137998$, side $=131908 \mathrm{Km} / \mathrm{sec}$ and amplify $=1.2$.

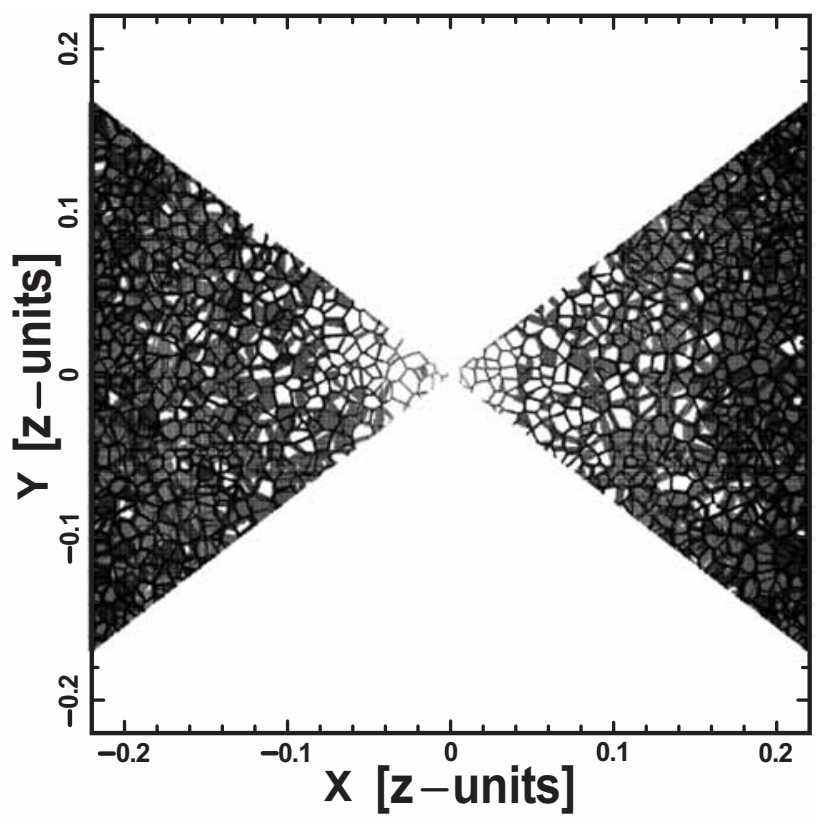

Fig. (11). The same as Fig. (10) but now two slices of $75^{\circ}$ long and $3^{\circ}$ wide are considered.

where $\Delta Z$ is the thickness of the slice and $\alpha$ is the opening angle, in our case $3^{\circ}$. 
In order to simulate the slices of observed galaxies a subset is extracted (randomly chosen) of the pixels belonging to a slice as represented, for example, in Fig. (11). In this operation of extraction of the galaxies from the pixels of the slice, the photometric rules as represented by formula (13) must be respected.

The cross sectional area of the VP can also be visualized through a spherical cut characterized by a constant value of the distance to the center of the box, in this case expressed in $z$ units, see Fig. (12) and Fig. (13); this intersection is called $V_{s}(2,3)$ where the index $s$ stands for sphere.

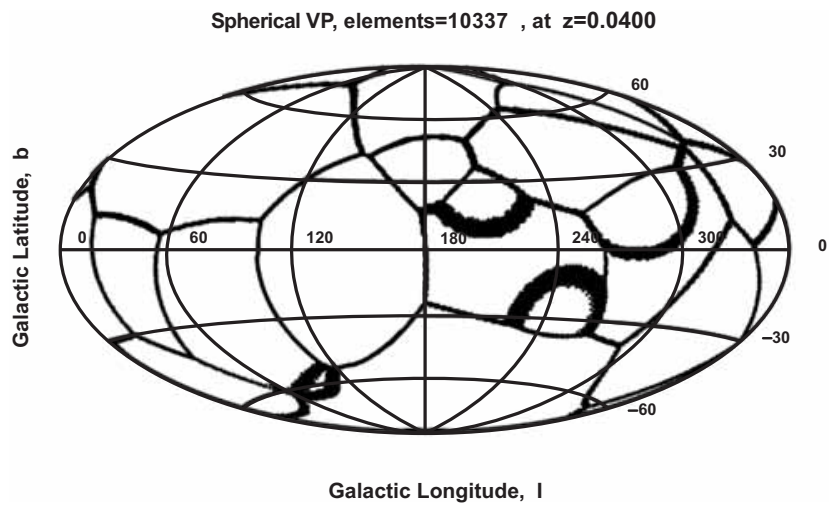

Fig. (12). The Voronoi--diagram $V_{s}(2,3)$ in the Hammer-Aitoff projection at $z=0.04$. The parameters are pixels $=400, N_{s}=$ 137998 , side $=131908 \mathrm{Km} / \mathrm{sec}$ and amplify $=1.2$.

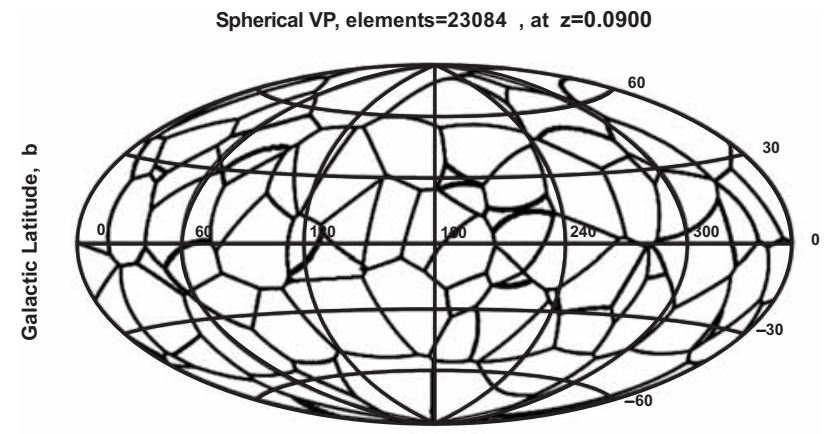

Galactic Longitude, I

Fig. (13). The Voronoi--diagram $V_{s}(2,3)$ in the Hammer-Aitoff projection at $z=0.09$; other parameters as in Fig. (12).

\section{THE STATISTICS OF THE VORONOI DIAGRAMS}

A probability density function (PDF) is the first derivative of a distribution function (DF) $F(x)$ with respect to $x$. In the case where the PDF is known but the DF is unknown, the following integral is evaluated

$$
F(x)=\int_{0}^{x} f(x) d x
$$

As a consequence the survival function (SF) is

$S F=1-F(x)$

We recall that the PVT is a particular case of the Voronoi tessellation in which the seeds are generated independently on the $X, Y$ and $Z$ axes in 3D.

\subsection{The Kiang Function}

The gamma variate $H(x ; c)$ ([19]) is

$H(x ; c)=\frac{c}{\Gamma(c)}(c x)^{c-1} \exp (-c x)$

where $0 \leq x<\infty, c>0$, and $\Gamma(c)$ is the gamma function with argument $c$. The Kiang PDF has a mean of

$\mu=1$

and variance

$\sigma^{2}=\frac{1}{c}$

This PDF can be generalized by introducing the dimension of the considered space, $d(d=1,2,3)$, and $c=2 d$

$H(x ; d)=\frac{2 d}{\Gamma(2 d)}(2 d x)^{2 d-1} \exp (-2 d x)$

In the case of a 1D PVT, $c=2$ is an exact analytical result and conversely $c$ is supposed to be 4 or 6 for 2D or 3D PVTs, respectively, [19]. The DF of the Kiang function, $\mathrm{DF}_{K}$, is

$D F_{K}=1-\frac{\Gamma(c, c x)}{\Gamma(c)}$

where the incomplete Gamma function, $\Gamma(a, z)$, is defined by

$\Gamma(a, z)=\int_{z}^{\infty} \mathrm{e}^{-t} t^{a-1} d t$

The survival function $S_{K}$ is

$S_{K}=\frac{\Gamma(c, c x)}{\Gamma(c)}$

\subsection{Generalized Gamma}

The generalized gamma PDF with three parameters $a, b, c,[76-78]$, is

$f(x ; b, c, d)=c \frac{b^{a / c}}{\Gamma(a / c)} x^{a-1} \exp \left(-b x^{c}\right)$

The generalized gamma has a mean of

$\mu=\frac{b^{-\frac{1}{c}} \Gamma\left(\frac{1+a}{c}\right)}{\Gamma\left(\frac{a}{c}\right)}$

and a variance of

$\sigma^{2}=\frac{b^{-\frac{2}{c}}\left(+\Gamma\left(\frac{2+a}{c}\right) \Gamma\left(\frac{a}{c}\right)-\left(\Gamma\left(\frac{1+a}{c}\right)\right)^{2}\right)}{\left(\Gamma\left(\frac{a}{c}\right)\right)^{2}}$

The SF of the generalized gamma is 


$$
S_{G G}=\frac{\Gamma\left(\frac{a}{c}, b x^{c}\right)}{\Gamma\left(\frac{a}{c}\right)}
$$

\subsection{Ferenc--Neda Function}

A new PDF has been recently introduced, [77], in order to model the normalized area/volume in a 2D/3D PVT

$$
F N(x ; d)=C \times x^{\frac{3 d-1}{2}} \exp (-(3 d+1) x / 2)
$$

where $C$ is a constant,

$$
C=\frac{\sqrt{2} \sqrt{3 d+1}}{22^{3 / 2 d}(3 d+1)^{-3 / 2 d} \Gamma(3 / 2 d+1 / 2)}
$$

and $d(d=1,2,3)$ is the dimension of the space under consideration. We will call this function the Ferenc--Neda PDF; it has a mean of

$\mu=1$

and variance

$\sigma^{2}=\frac{2}{3 d+1}$

The SF of the Ferenc--Neda function when $d=3$ is

$S_{F N 3}=\mathrm{e}^{-5 x}+5 \mathrm{e}^{-5 x} x$

$+\frac{25}{2} \mathrm{e}^{-5 x} x^{2}+\frac{125}{6} \mathrm{e}^{-5 x} x^{3}+\frac{625}{24} x^{4} \mathrm{e}^{-5 x}$

\subsection{Kiang Function of the Radius}

We now analyze the distribution in effective radius $R$ of the 3D PVT. We assume that the volume of each cell, $v$, is

$v=\frac{4}{3} \pi\left(\frac{R}{\rho}\right)^{3}$,

where $\rho$ is a length that connects the normalized radius to the observed one. In the following, we derive the PDF for radius and related quantities relative to the Kiang function and Ferenc--Neda function. The PDF, $H_{R}(R ; c)$, of the radius corresponding to the Kiang function as represented by (44) is

$$
H_{R}(R ; c)=\frac{4 c\left(4 / 3 \frac{c \pi R^{3}}{\rho^{3}}\right)^{c-1} e^{-4 / 3 \frac{c \pi R^{3}}{\rho^{3}}} \pi R^{2}}{\Gamma(c) \rho^{3}}
$$

where $0 \leq R<\infty, c>0$ and $\rho>0$. The Kiang PDF of the radius has a mean of

$$
\mu=1 / 2 \frac{\sqrt[3]{2} \sqrt[3]{3} \Gamma(1 / 3+c)}{\sqrt[3]{c} \sqrt[3]{\pi} \Gamma(c)} \rho
$$

and variance $\sigma^{2}=\frac{1}{4} \frac{3^{\frac{2}{3}} 2^{\frac{2}{3}}\left(\Gamma(2 / 3+c) \Gamma(c)-(\Gamma(1 / 3+c))^{2}\right)}{c^{2 / 3} \pi^{2 / 3}(\Gamma(c))^{2}} \rho^{2}$

The survival function of the Kiang function in radius is

$$
S_{K R}=\frac{\Gamma\left(c, 4 / 3 c \pi\left(\frac{R}{\rho}\right)^{3}\right)}{\Gamma(c)}
$$

Table 4. Values of $x^{2}$ for the Cell Normalized Area-Distribution Function of $V_{p}(2,3)$; here $T_{i}$ are the Theoretical Frequencies and $O_{i}$ are the Sample Frequencies. Here we have 8517 Poissonian Seeds and 40 Intervals in the Histogram

\begin{tabular}{|c|c|}
\hline PDF & Parameter $\boldsymbol{x}^{2}$ \\
\hline \hline $\mathrm{H}(x ; c)$ Eq.()) $\mathrm{c}=2.07$ & 114.41 \\
\hline $\mathrm{P}(x ; b)$ Eq.()) $\mathrm{d}=1$ & 85.38 \\
\hline
\end{tabular}

\subsection{The Ferenc--Neda Function of the Radius}

The PDF as a function of the radius, obtained from (55) and inserting $d=3$, is

$F N_{R}(R ; d)=\frac{400000 \pi^{5} R^{14} e^{-\frac{20}{3} \frac{\pi R^{3}}{\rho^{3}}}}{243 \rho^{15}}$

The mean of the Ferenc--Neda function is

$\mu=0.6 \rho$

and the variance is

$\sigma^{2}=0.0085 \rho^{2}$

The SF of the Ferenc--Neda function of the radius when $d=3$ is

$$
\begin{aligned}
& S_{F N 3 R}=e^{-\frac{20}{3} \frac{\pi R^{3}}{\rho^{3}}}+\frac{20}{3} e^{-\frac{20}{3} \frac{\pi R^{3}}{\rho^{3}}} R^{3} \pi \rho^{-3}+\frac{200}{9} e^{-\frac{20}{3} \frac{\pi R^{3}}{\rho^{3}}} R^{6} \pi^{2} \rho^{-6} \\
& +\frac{4000}{81} e^{-\frac{20}{3} \frac{\pi R^{3}}{\rho^{3}}} R^{9} \pi^{3} \rho^{-9}+\frac{20000}{243} e^{-\frac{20}{3} \frac{\pi R^{3}}{\rho^{3}}} R^{12} \pi^{4} \rho^{-12}
\end{aligned}
$$

\subsection{Kiang Distribution of $V_{p}(2,3)$ in Radius}

Here, we first model the normalized area-distribution $V_{p}(2,3)$ with Kiang PDFs as represented by (44), see Table 4.

The PDF, $H_{R 23}(R ; c)$, as a function of the radius corresponding to the Kiang function as represented by (44) for $V_{p}(2,3)$ is

$H_{R 23}(R ; c)=\frac{2 c\left(\frac{c \pi R^{2}}{\rho^{2}}\right)^{c-1} \mathrm{e}^{-\frac{c \pi R^{2}}{\rho^{2}}} \pi R}{\Gamma(c) \rho^{2}}$ 
where $0 \leq R<\infty, c>0$ and $\rho>0$. The Kiang PDF of the radius for $V_{p}(2,3)$ has a mean of

$\mu=\frac{\rho \Gamma(c+1 / 2)}{\sqrt{c} \sqrt{\pi} \Gamma(c)}$

and variance

$$
\sigma^{2}=\frac{\rho^{2}\left(c(\Gamma(c))^{2}-(\Gamma(c+1 / 2))^{2}\right)}{c \pi(\Gamma(c))^{2}}
$$

The survival function of the Kiang function , $S_{K R 23}$, in radius for $V_{p}(2,3)$ is

$S_{K R 23}=\frac{\Gamma\left(c, 2 \frac{c \pi R^{2}}{\rho^{2}}\right)}{\Gamma(c)}$

A comparison of the survival function $S_{K R 23}$ of the radius and the exponential distribution is reported in Fig. (14).

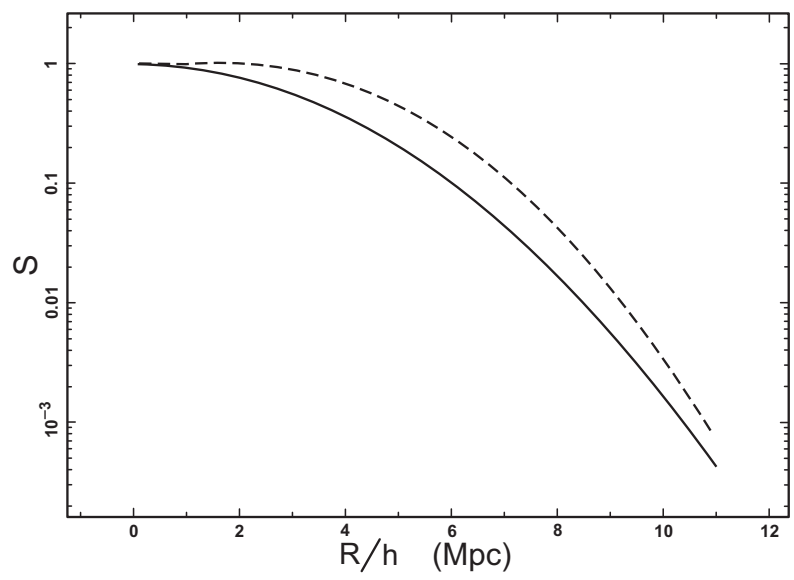

Fig. (14). The survival function, $S_{K R 23}$, of the radius of the Kiang function for $V_{p}(2,3)$ as represented by (72) when $\rho=13 \mathrm{Mpc}$, $c=2.25$ and $\chi^{2}=67.1$ with 100 points (dashed line). The survival function, $S_{K R 23}$, of the radius of the exponential distribution for $V_{p}(2,3)$ as represented by (78) when $\rho=7 \mathrm{Mpc}$ and $\chi^{2}=9.27$ with 100 points (full line).

\subsection{Exponential Distribution of $V_{p}(2,3)$ in Radius}

Another PDF that can be considered in order to model the normalized area distribution of $V_{p}(2,3)$ is the exponential distribution,

$$
p(x)=\frac{1}{b} \exp -\frac{x}{b}
$$

which has an average value

$$
\bar{x}=b
$$

In the case of the normalized areas $b=1$, Table 4 reports the $\chi^{2}$ values of the two distributions adopted here. The
PDF, $P_{R 23}(R ; c)$, as a function of the radius corresponding to the exponential distribution as represented by (73) for $V_{p}(2,3)$ is

$p_{R 23}(R ; c)=\frac{2 \mathrm{e}^{-\frac{\pi R^{2}}{\rho^{2}}} \pi R}{\rho^{2}}$

where $0 \leq R<\infty, \rho>0$. The exponential PDF of the radius for $V_{p}(2,3)$ has a mean of

$\mu=\frac{\pi^{3 / 2}}{2 \rho^{2}\left(\frac{\pi}{\rho^{2}}\right)^{3 / 2}}$

and variance

$\sigma^{2}=\frac{\rho^{2}(4-\pi)}{4 \pi}$

The survival function of the exponential distribution , $S_{E R 23}$, in radius for $V_{p}(2,3)$ is

$S_{E R 23}=\mathrm{e}^{-\frac{\pi R^{2}}{\rho^{2}}}$

Fig. (14) reports a comparison between the survival function of Kiang distribution and the exponential distribution for $V_{p}(2,3)$. More details can be found in [79].

\section{STEREOLOGY}

We first briefly review how a PDF $f(x)$ changes to $g(y)$ when a new variable $y(x)$ is introduced. We limit ourselves to the case in which $y(x)$ is a one-to-one transformation. The rule for transforming a PDF is

$$
g(y)=\frac{f(x)}{\left|\frac{d y}{d x}\right|}
$$

Analytical results have shown that sections through Ddimensional Voronoi tessellations are not themselves D-1 Voronoi tessellations, see [80-82]. According to [83], the probability of a plane intersecting a given sphere is proportional to the sphere's radius, $R$. Cross-sections of radius $r$ may be obtained from any sphere with a radius greater than or equal to $r$. We may now write a general expression for the probability of obtaining a cross-section of radius $r$ from the whole distribution (which is denoted $F(R))$ :

$f(r)=\int_{r}^{\infty} F(R) R \frac{1}{R} \frac{r}{\sqrt{R^{2}-r^{2}}} d R$

which is formula (A7) in [83]. That is to say, $f(r)$ is the probability of finding a bubble of radius $R$, multiplied by the probability of intersecting this bubble, multiplied by the probability of obtaining a slice of radius $r$ from this bubble, integrated over the range of $R \geq r$. A first example is given by the so called monodisperse bubble size distribution (BSD) which are bubbles of constant radius $R$ and therefore 


$$
F(R)=\frac{1}{R}
$$

which is defined in the interval $[0, R]$ and

$$
f(r)=\frac{r}{\sqrt{R^{2}-r^{2}} R}
$$

which is defined in the interval $[0, R]$, see Eq. (A4) in [83]. The average value of the radius of the $2 \mathrm{D}$-slices is

$\bar{r}=1 / 4 R \pi$

the variance is

$\sigma^{2}=2 / 3 R^{2}-1 / 16 R^{2} \pi^{2}$

and finally,

Skewness $=-1.151, \quad$ Kurtosis $=0.493$.

\subsection{PVT Stereology}

In order to find our $F(R)$, we now analyze the distribution in effective radius $R$ of the 3D PVT. We assume that the volume of each cell, $v$, is

$v=\frac{4}{3} \pi R^{3}$

In the following, we derive the PDF for the radius and related quantities relative to the Ferenc--Neda function. The $\mathrm{PDF}$ as a function of the radius according to the rule of change of variables (79), is obtained from (55) on inserting $d=3$ and was already defined, see equation 65 . The average radius is

$$
\bar{R}=0.6065
$$

Table 5. The Parameters of f(r), Eq. (91), Relative to the PVT Case

\begin{tabular}{|c|c|}
\hline Parameter & Value \\
\hline \hline Mean & 0.4874 \\
\hline Variance & 0.02475 \\
\hline Mode & 0.553 \\
\hline Skewness & -.5229 \\
\hline Kurtosis & -.1115 \\
\hline
\end{tabular}

and the variance is

$\sigma^{2}(R)=0.00853$

The introduction of the scale factor, $b$, with the new variable $R=R^{\prime} / b$ transforms Eq. (65) into

$F\left(R^{\prime}, b\right)=\frac{400000 \pi^{5} R^{14} \mathrm{e}^{-\frac{20}{3} \frac{\pi R^{3}}{b^{3}}}}{243 b^{15}}$
We now have $F(R)$ as given by Eq. (65) and the fundamental integral (80), as derived in [84], is

$f(r)=2 / 3 K \sqrt[6]{3} \sqrt[3]{10} \sqrt[3]{\pi} r G_{3,5}^{4,1}\left(\left.\frac{100}{9} \pi^{2} r^{6}\right|_{7 / 3,2 / 3,1 / 3,0, \frac{17}{6}} ^{5 / 6,1 / 6,1 / 2}\right)$

$0 \leq r \leq 1$

where $K$ is a constant,

$K=1.6485$

and the Meijer $G$-function is defined as in [85-87].Details on the real or complex parameters of the Meijer $G$-function are given in the Appendix of [88]. Table 5 shows the average value, variance, mode, skewness, and kurtosis of the already derived $f(r)$.

Asymptotic series are

$f(r) \sim 2.7855 r$

when $\quad r \rightarrow 0$,

and

$f(r) \sim-0.006(r-1)+0.136(r-1)^{2}$

when $\quad r \rightarrow 1$

The distribution function (DF) is

$D F(r)=$

$\frac{1}{90} K 3^{5 / 6} 10^{2 / 3} G_{4,6}^{4,2}\left(\left.\frac{100}{9} \pi^{2} r^{6}\right|_{8 / 3,1,2 / 3,1 / 3, \frac{19}{6}, 0} ^{1,7 / 6,1 / 2,5 / 6}\right) \frac{1}{\sqrt[3]{\pi}}$

$0 \leq r \leq 1$

The already defined PDF is defined in the interval $0 \leq r \leq 1$. In order to make a comparison with a normalized sample which has a unitarian mean or an astronomical sample which has the mean expressed in Mpc, a transformation of scale should be introduced. The change of variable is $r=x / b$ and the resulting PDF is

Table 6. Parameters of $f(x, b)$, Eq. (96), Relative to the PVT Case

\begin{tabular}{|c|c|c|}
\hline Parameter & $\mathbf{b}=\mathbf{2 . 0 5 1}$ & $\mathbf{b}=\mathbf{3 4}$ \\
\hline \hline Mean & 1. & $16.57 \mathrm{Mpc}$ \\
\hline Variance & 0.104 & $28.62 \mathrm{Mpc}^{2}$ \\
\hline Mode & 1.134 & $18.80 \mathrm{Mpc}^{2}$ \\
\hline
\end{tabular}

Table 7. The Values of $\chi^{2}$ for the Cell Normalized AreaDistribution of $V_{p}(2,3)$. The Number of 2D Cells is 789, the 3D Seeds are 15000 and the Number of Bins in the Histogram is 30

\begin{tabular}{|c|c|c|}
\hline PDF & Parameter & $\boldsymbol{x}^{2}$ \\
\hline \hline$H(x ; c)($ Eq.()) & $\mathrm{c}=5.8$ & 250.8 \\
\hline
\end{tabular}




\begin{tabular}{|c|c|c|}
\hline$f(x ; c)$ (Eq.()) & $\mathrm{d}=5.8$ & 250.8 \\
\hline$f(x ; c)$ (Eq.)) & $\mathrm{b}=0.0514$ & 127 \\
\hline
\end{tabular}

$f(x, b)=$

$\frac{2}{3} K \sqrt[6]{3} \sqrt[3]{10} \sqrt[3]{\pi} x G_{3,5}^{4,1}\left(\left.\frac{100}{9} \frac{\pi^{2} x^{6}}{b^{6}}\right|_{7 / 3,2 / 3,1 / 3,0, \frac{17}{6}} ^{5 / 6,1 / 6,1 / 2}\right)\left(\frac{1}{b}\right)^{2}$

$0 \leq r \leq b$

As an example, Table 6 shows the statistical parameters for two different values of $b$. Skewness and kurtosis do not change with a transformation of scale.

We briefly recall that a PDF $f(x)$ is the first derivative of a distribution function (DF) $F(x)$ with respect to $x$. When the DF is unknown but the PDF known, we have

$F(x)=\int_{0}^{x} f(x) d x$

The survival function (SF) $S(x)$ is

$S(x)=1-F(x)$

and represents the probability that the variate takes a value greater than $x$. The SF with the scaling parameter $b$ is

$$
\begin{aligned}
& S F(x, b)= \\
& 1-0.018313^{5 / 6} 10^{2 / 3} G_{4,6}^{4,2}\left(\left.\frac{100}{9} \frac{x^{6} \pi^{2}}{b^{6}}\right|_{8 / 3,1,2 / 3,1 / 3, \frac{19}{6}, 0} ^{1,7 / 6,1 / 2,5 / 6}\right) \frac{1}{\sqrt[3]{\pi}}
\end{aligned}
$$

$0 \leq r \leq b$.

A first application can be a comparison between the real distribution of radii of $V_{p}(2,3)$, see Fig. (15), and the already obtained rescaled $\operatorname{PDF} f(x, b)$. The fit with the rescaled $f(x, b)$ is shown in Fig. (16) and Table 7 shows the $\chi^{2}$ of three different fitting functions.

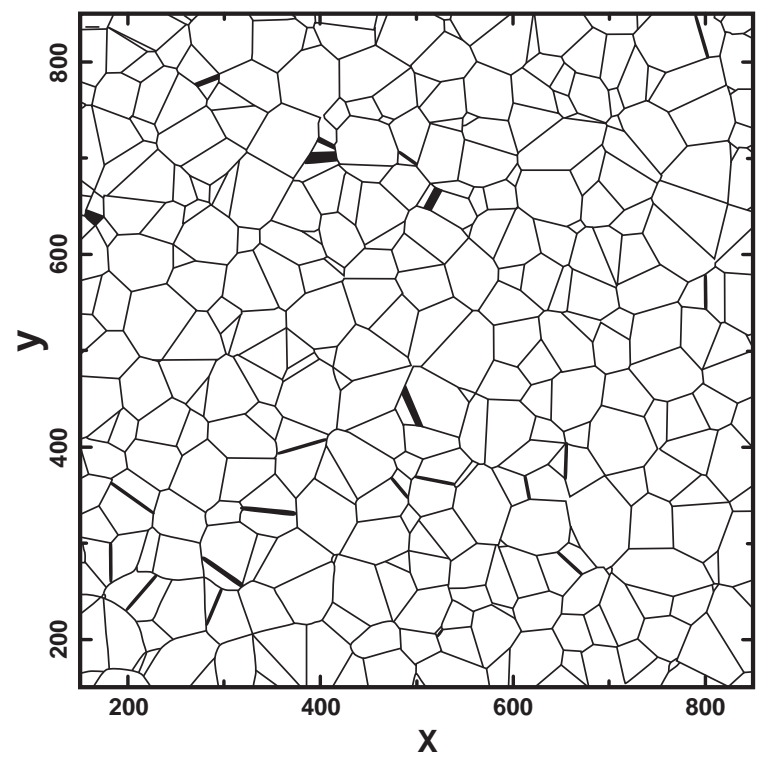

Fig. (15). PVT diagram $\operatorname{Vp}(2,3)$ when 789 2D cells generated by 15000 3D seeds are considered.

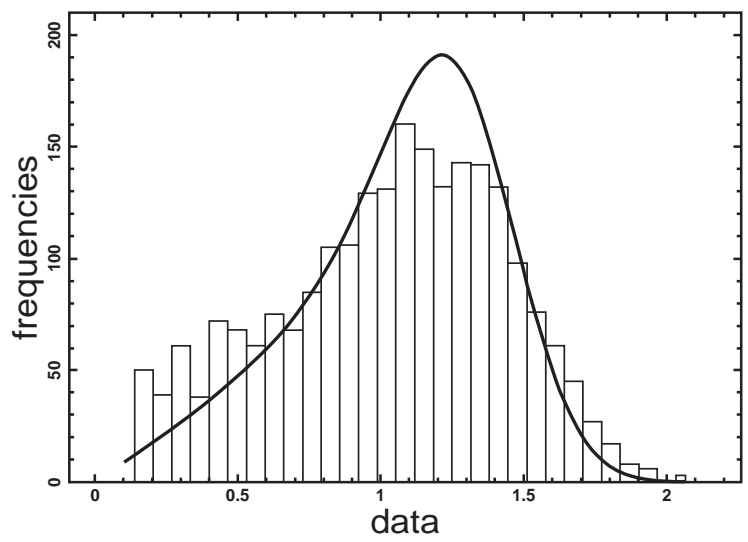

Fig. (16). Histogram (step-diagram) of PVT $V_{p}(2,3)$ when $7892 \mathrm{D}$ cells, generated by 15000 3D seeds, are considered. The superposition of the $f(x, b)$, Eq. (100), is displayed.

The PDF $f_{A}$ of the areas of $V_{p}(2,3)$ can be obtained from $f(r)$ by means of the transformation, see [84],

$f_{A}(A)=f(r)\left(\left(\frac{A}{\pi}\right)^{1 / 2}\right) \frac{\pi^{-1 / 2}}{2} A^{-1 / 2}$

that is,

$f_{A}(A)=0.549 \sqrt[6]{3} \sqrt[3]{10} G_{3,5}^{4,1}\left(\left.\frac{100}{9} \frac{A^{3}}{\pi}\right|_{7 / 3,2 / 3,1 / 3,0, \frac{17}{6}} ^{5 / 6,1 / 6,1 / 2}\right) \pi^{-2 / 3}$

The already derived $f_{A}(A)$ has average value, variance, mode, skewness and kurtosis as shown in Table 8 .

Table 8. Parameters of $f_{A}(A)$, Eq. (100), Relative to the PVT Case

\begin{tabular}{|c|c|}
\hline Parameter & Value \\
\hline \hline Mean & 0.824 \\
\hline Variance & 0.204 \\
\hline Mode & 0.858 \\
\hline Skewness & 0.278 \\
\hline Kurtosis & -.0 .337 \\
\hline
\end{tabular}

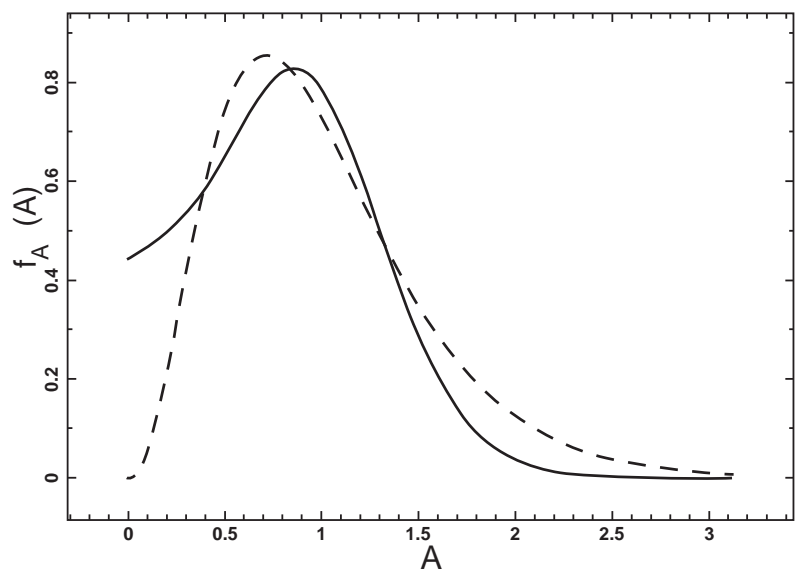

Fig. (17). The $\operatorname{PDF} f_{A}$, Eq. (100), as a function of A (full line) and $\mathrm{FN}(\mathrm{x} ; \mathrm{d})$, Eq. (55), when $\mathrm{d}=2$ (dotted line). 
Since, for $r$ close to $0, f(r) \sim r$ from Eq. (100) it follows that $f_{A}(0) \neq 0$, in particular $f_{A}(0)=0.443$ and Fig. (17) shows the graph of $f_{A}$.

The previous figure shows that sections through 3dimensional Voronoi tessellations are not themselves 2dimensional Voronoi tessellations because $f_{A}(0)$ has a finite value rather than 0 as does the $2 \mathrm{D}$ area distribution; this fact can be considered a numerical demonstration in agreement with [82]. The distribution function $F_{A}$ is given by

$$
F_{A}=0.0183^{5 / 6} 10^{2 / 3} G_{4,6}^{4,2}\left(\left.\frac{100}{9} \frac{A^{3}}{\pi}\right|_{8 / 3,1,2 / 3,3,1 /, \frac{19}{6}, 0} ^{1,7 / 6,1 / 2,5}\right) \frac{1}{\sqrt[3]{\pi}}
$$

Consider a three-dimensional Poisson Voronoi diagram and suppose it intersects a randomly oriented plane $\gamma$ : the resulting cross sections are polygons.

A comparison between $F_{A}$ and the area of the irregular polygons is shown in Fig. (18). In this case the number of seeds is 300000 and we processed 100168 irregular polygons obtained by adding together results of cuts by 41 triples of mutually perpendicular planes. The maximum distance between the two curves is $d_{\max }=0.039$.

As concerns the linear dimension, in our approximation the two-dimensional cells were considered circles and thus, for consistency, the radius $r$ of an irregular polygon was defined as

$r=\left(\frac{A}{\pi}\right)^{1 / 2}$

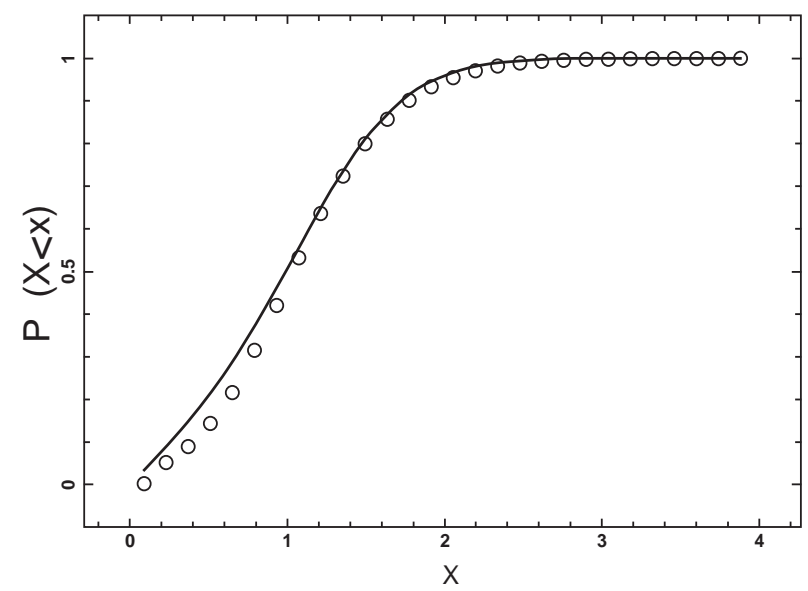

Fig. (18). Comparison between data (empty circles) and theoretical curve (continuous line) of the distribution of areas of the planar cross sections.

that is, $r$ is the radius of a circle with the same area, $A$, as the polygon. The assumption of sphericity can be considered an axiom of the theory here presented, but for a more realistic situation the stereological results will be far more complex. More details can be found in [88].

\subsection{NPVT Stereology}

An example of NPVT is represented by a distribution in volume which follows a Kiang function as given by Eq. (44). The case of PVT volumes indicates $c=5$, or $c=6$, the so called Kiang conjecture: we will take $c$ as a variable. The resulting distribution in radius once the scaling parameter $b$ is introduced is

$F_{K}(R, b, c)=\frac{4 c\left(4 / 3 \frac{c \pi R^{3}}{b^{3}}\right)^{c-1} \mathrm{e}^{-4 / 3 \frac{c \pi R^{3}}{b^{3}}} \pi R^{2}}{\Gamma(c) b^{3}}$

The average radius is

$\bar{R}=\frac{\sqrt[3]{2} \sqrt[3]{3} b \Gamma(1 / 3+c)}{2 \sqrt[3]{c} \sqrt[3]{\pi} \Gamma(c)}$

and the variance is

$\sigma^{2}(R)=\frac{-3^{2 / 3} 2^{2 / 3} b^{2}\left(-\Gamma(2 / 3+c) \Gamma(c)+(\Gamma(1 / 3+c))^{2}\right)}{4 c^{2 / 3} \pi^{2 / 3}(\Gamma(c))^{2}}$

The skewness is

$$
\gamma=\frac{(\Gamma(c))^{3} c-3 \Gamma(c) \Gamma(1 / 3+c) \Gamma(2 / 3+c)+2(\Gamma(1 / 3+c))^{3}}{\left(\Gamma(2 / 3+c) \Gamma(c)-(\Gamma(1 / 3+c))^{2}\right)^{3 / 2}}
$$

and the kurtosis is given by a complicated analytical expression. Fig. (19) shows a superposition of the effective radii of the voids in SDSS DR7 with a superposition of the curve of the theoretical PDF in the radius, $F_{K}(R, b, c)$, as represented by Eq. (103). Table 9 shows the theoretical statistical parameters.

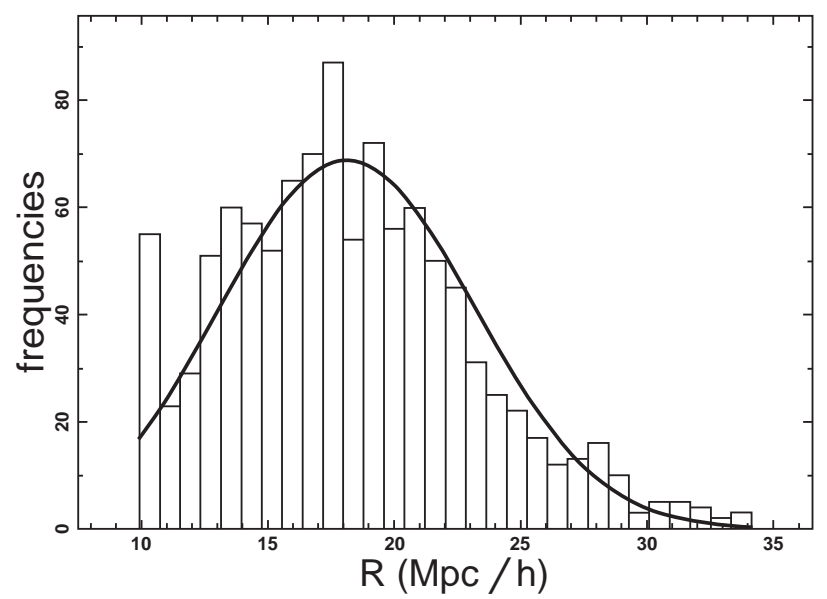

Fig. (19). Figure 19. Histogram (step-diagram) of the effective radius in the SDSS DR7 with a superposition of the PDF in radius of the NPVT spheres, FK(R, b, c), as represented by Eq. (103). The number of bins is $30, b=31.33 \mathrm{Mpc}$, and $\mathrm{c}=1.768$.

Table 9. The Values of $\chi^{2}$ for the Cell Normalized AreaDistribution of $V_{p}(2,3)$. The Number of $2 \mathrm{D}$ cells is 789 , the 3D Seeds are 15000 and the Number of Bins in the Histogram is 30

\begin{tabular}{|c|c|}
\hline Parameter & Value \\
\hline \hline Mean & $18.23 \mathrm{~h}^{-1} \mathrm{Mpc}$ \\
\hline Variance & $23.31 \mathrm{~h}^{-2} \mathrm{Mpc}^{2}$ \\
\hline
\end{tabular}




\begin{tabular}{|c|c|}
\hline Standard deviation & $4.82 \mathrm{~h}^{-1} \mathrm{Mpc}$ \\
\hline Skewness & 0.072 \\
\hline Kurtosis & -0.162 \\
\hline
\end{tabular}

Table 10. NPVT Parameters of $f(r)_{N P V T K}$, Eq. (108)

\begin{tabular}{|c|c|}
\hline Parameter & Value \\
\hline \hline Mean & 0.488 \\
\hline Variance & 0.0323 \\
\hline Mode & 0.517 \\
\hline Skewness & -.114 \\
\hline Kurtosis & 2.614 \\
\hline
\end{tabular}

The result of the integration of the fundamental Eq. (80) inserting $c=2$ gives the following PDF for the radius of the cuts

$$
f(r)_{N P V T K}=3.4148 \sqrt[6]{3} \sqrt[3]{\pi} r 2^{2 / 3} G_{2,4}^{4,0}\left(\left.\frac{16}{9} \pi^{2} r^{6}\right|_{4 / 3,2 / 3,1 / 3,0} ^{1 / 6,1 / 2}\right)
$$

$0 \leq r \leq 1$ 10.

The statistics of NPVT cuts with $c=2$ are shown in Table

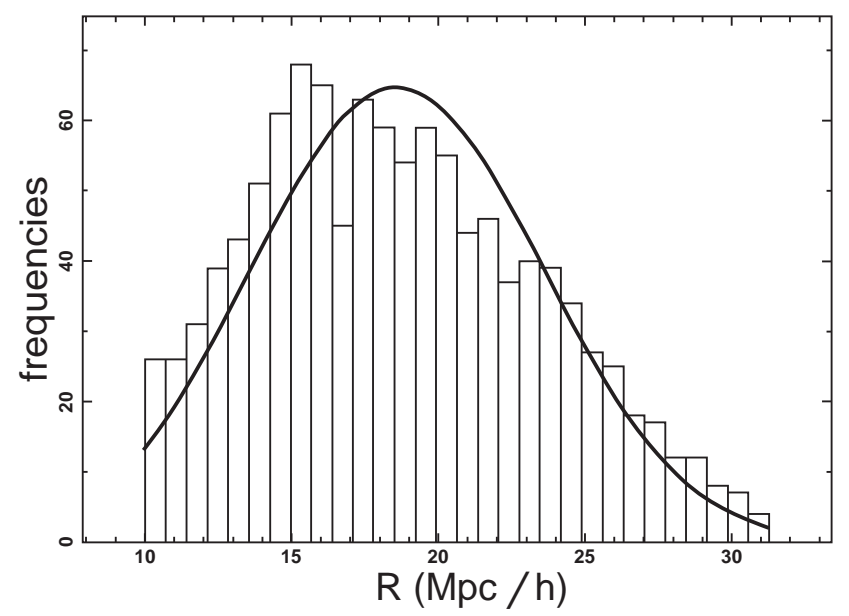

Fig. (20). Figure 20. Histogram (step-diagram) of the simulated effective radius of SDSS DR7 with a superposition of the PDF in radius of the PVT spheres as represented by Eq. (103). The artificial sample has a minimum value of $10 / \mathrm{h} \mathrm{Mpc}$, the number of bins is 30 , $\mathrm{b}=31.5 / \mathrm{h} \mathrm{Mpc}$, and $\mathrm{c}=1.3$.

On introducing the scaling parameter $b$, the PDF which describes the radius of the cut becomes

$f(x, b)_{N P V T K}=3.4148 \sqrt[6]{3} \sqrt[3]{\pi} x 2^{2 / 3} G_{2,4}^{4,0}\left(\left.\frac{16}{9} \frac{\pi^{2} x^{6}}{b^{6}}\right|_{4 / 3,2 / 3,1 / 3,0} ^{1 / 6,12}\right) b^{-2}$

$0 \leq r \leq b$

The SF of the second NPVT case, $S F_{N P V T K}$, with the scaling parameter $b$, is

$S F(x, b)_{N P V T K}=1-0.28453 \sqrt[3]{5 / 6} G_{3,5}^{4,1}\left(\left.\frac{16}{9} \frac{\pi^{2} x^{6}}{b^{6}}\right|_{5 / 3,1,2 / 3,1 / 3,0} ^{1,1 / 2,5 / 6}\right) \frac{1}{\sqrt[3]{\pi}}$
$0 \leq r \leq b$

A careful exploration of the distribution in effective radius of SDSS DR7 reveals that the detected voids have radius $\geq 10 / \mathrm{h} \mathrm{Mpc}$. This observational fact demands the generation of random numbers in the distribution in radii of the $3 \mathrm{D}$ cells as given by Eq. (103) with a minimal value of $10 / \mathrm{h} \mathrm{Mpc}$. The artificial sample is generated through a numerical computation of the inverse function [89] and displayed in Fig. (20); the sample's statistics are shown in Table 11.

More details can be found in [88].

\section{THE CELlULAR STRUCTURE OF THE UNIVERSE}

From a simplified point of view the galaxies belonging to a given catalog are characterized by the astronomical coordinates, the redshift and the apparent magnitude. Starting from the second CFA2 redshift Survey, the catalogs were organized in slices of a given opening angle, $3^{\circ}$ or $6^{\circ}$, and a given angular extension, for example $130^{\circ}$. When plotted in polar coordinates of $c_{l} z$ the spatial distribution of galaxies is not random but distributed on filaments. Particular attention should be paid to the fact that the astronomical slices are not a plane which intersects a Voronoi Network. In order to quantify this effect we introduce a confusion distance, $D V_{c}$, as the distance after which the half altitude of the slices equalizes the observed average diameter $D V^{o b s}$

Table 11. The Statistical Parameters of the Artificially Generated Radius with a Lower Bound of $10 / \mathrm{h} \mathrm{Mpc}, \mathrm{c}=1.3$

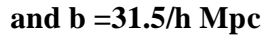

\begin{tabular}{|c|c|}
\hline Parameter & Value \\
\hline \hline Mean & $18.69 \mathrm{~h}^{-1} \mathrm{Mpc}$ \\
\hline Variance & $22.74 \mathrm{~h}^{-2} \mathrm{Mpc}^{2}$ \\
\hline Standard deviation & $4.76 \mathrm{~h}^{-1} \mathrm{Mpc}$ \\
\hline Skewness & 0.33 \\
\hline Kurtosis & -0.623 \\
\hline Maximum value & $31.27 \mathrm{~h}^{-1} \mathrm{Mpc}$ \\
\hline Minimum value & $10 \mathrm{~h}^{-1} \mathrm{Mpc}$ \\
\hline
\end{tabular}

$D V_{c} \tan (\alpha)=\frac{1}{2} \overline{D V^{o b s}}$

where $\alpha$ is the opening angle of the slice and $D V^{\text {obs }}$ the averaged diameter of voids. In the case of 2dFGRS $\alpha=3^{\circ}$ and therefore $D V_{c}=2.5710^{4} \frac{\mathrm{Km}}{\mathrm{sec}}$ when $\overline{D V^{o b s}}=2700 \frac{\mathrm{Km}}{\mathrm{sec}}$.

For values of $c_{l} z$ greater than $D V_{c}$ the voids in the distribution of galaxies are dominated by the confusion. For values of $c_{l} z$ lower than $D V_{c}$ the filaments of galaxies can be considered the intersection between a plane and the faces of the Voronoi Polyhedrons. A measure of the portion of the sky covered by a catalog of galaxies is the area covered by a 
unitarian sphere which is $4 \pi$ steradians or $\frac{129600}{\pi}$ square degrees. In the case of $2 \mathrm{dFGRS}$ the covered area of two slices of $75^{\circ}$ long and $3^{\circ}$ wide, as in Fig. (23), is $\frac{1414}{\pi}$ square degrees or $0.13 \mathrm{sr}$. In the case of RC3 the covered area it is $4 \pi$ steradians with the exclusion of the Zone of Avoidance, see Fig. (2). In the following we will simulate the 2dFGRS, a catalog that occupies a small area of the sky, the RC3 a catalog that occupies all the sky and the CFA2 catalog.

In the case of $3 \mathrm{C} 3$ we demonstrate how it is possible to simulate the Zone of Avoidance in the theoretical simulation. The paragraph ends with a discussion on the Eridanus supervoid also known as "Cold Spot".

\subsection{The 2dFGRS}

Fig. (21) shows the galaxies of the 2dFGRS with $z<0.3$ in galactic coordinates and the two strips in the 2dFGRS are shown in Fig. (22).

Fig. (23) conversely reports the 2dfGRS catalog when a slice of $75^{\circ} \times 3^{\circ}$ is taken into account. This slice represents the object to simulate.

The previous observational slice can be simulated by adopting the Voronoi network reported in Fig. (11).

The distribution of the galaxies as given by the Voronoi Diagrams is reported in Fig. (24) where all the galaxies are considered. In this case the galaxies are extracted according to the integral of the Schechter function in flux (formula (15) with parameters as in Table 2). Table 12 reports the basic data of the astronomical and simulated data of the $75^{\circ} \times 3^{\circ}$ slice.

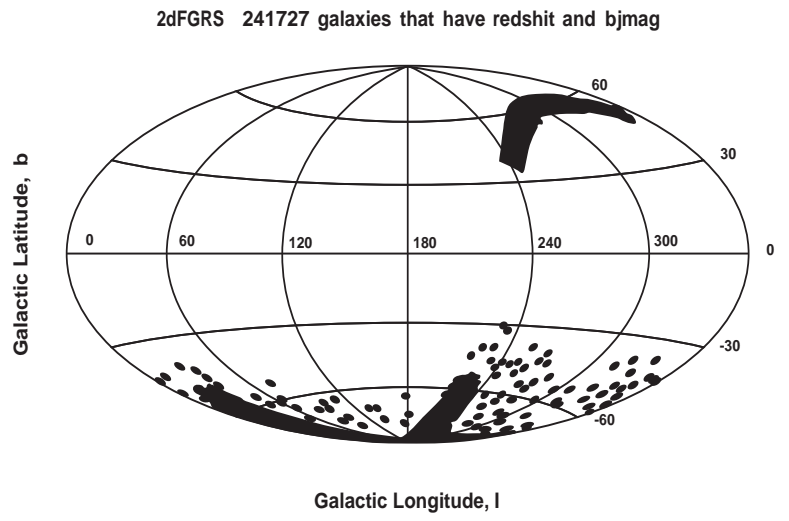

Fig. (21). Hammer-Aitoff projection in galactic coordinates of 230540 galaxies in the 2dfGRS which have bJmag and redshift < 0.3 .

When conversely a given interval in flux (magnitudes) characterized by $f_{\min }$ and $f_{\max }$ is considered the number of galaxies, $N_{S C}$, of a $3^{\circ}$ slice can be found with the following formula

$$
N_{S C}=N_{C} \frac{\int_{f_{\min }}^{f_{\max }} 4 \pi\left(\frac{c_{l}}{H_{0}}\right)^{5} z^{4} \Phi\left(\frac{z^{2}}{z_{\text {crit }}^{2}}\right) d f}{\int_{f_{\min , C}, C}^{f_{\max }} 4 \pi\left(\frac{c_{l}}{H_{0}}\right)^{5} z^{4} \Phi\left(\frac{z^{2}}{z_{\text {crit }}^{2}}\right) d f}
$$

where $f_{\min , C}$ and $f_{\max , C}$ represent the minimum and maximum flux of the considered catalog and $N c$ all the galaxies of the considered catalog; a typical example is reported in Fig. (25).

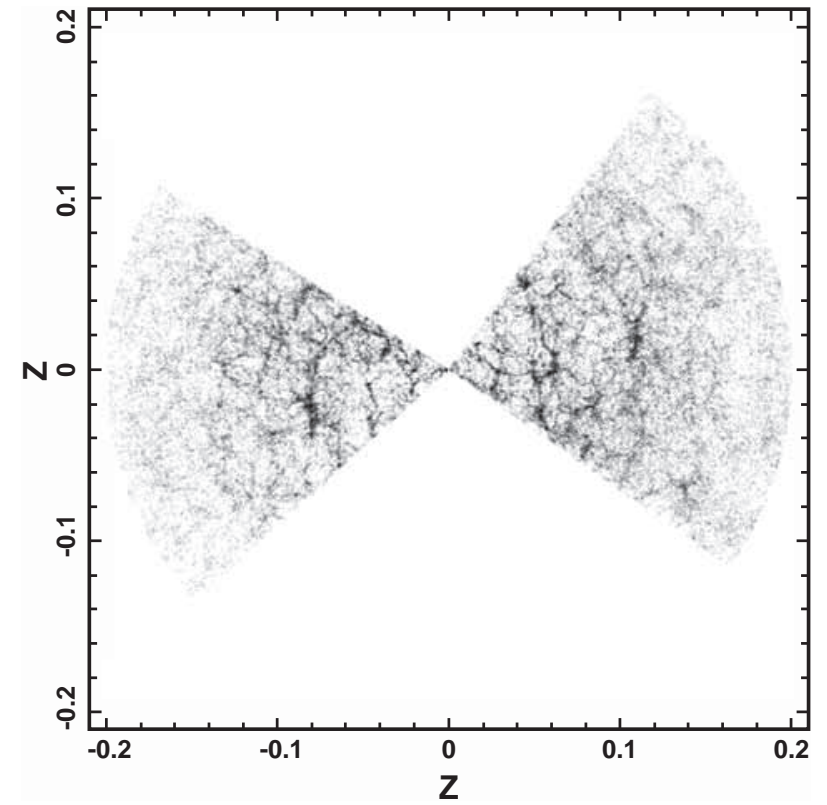

Fig. (22). Cone-diagram of all the galaxies in the 2dFGRS. This plot contains 203249 galaxies.

Table 12. Real and Simulated data of the slice $75^{\circ}$ long and $3^{\circ}$ wide

\begin{tabular}{|c|c|c|}
\hline & 2dFGRS & Simulation \\
\hline Elements & $\mathbf{6 2 5 5 9}$ & $\mathbf{6 2 5 6 3}$ \\
\hline \hline$Z_{\min }$ & 0.001 & 0.011 \\
\hline$Z_{\text {pos-max }}$ & 0.029 & 0.042 \\
\hline$Z_{\text {ave }}$ & 0.0651 & 0.058 \\
\hline$Z_{\text {max }}$ & 0.2 & 0.2 \\
\hline
\end{tabular}

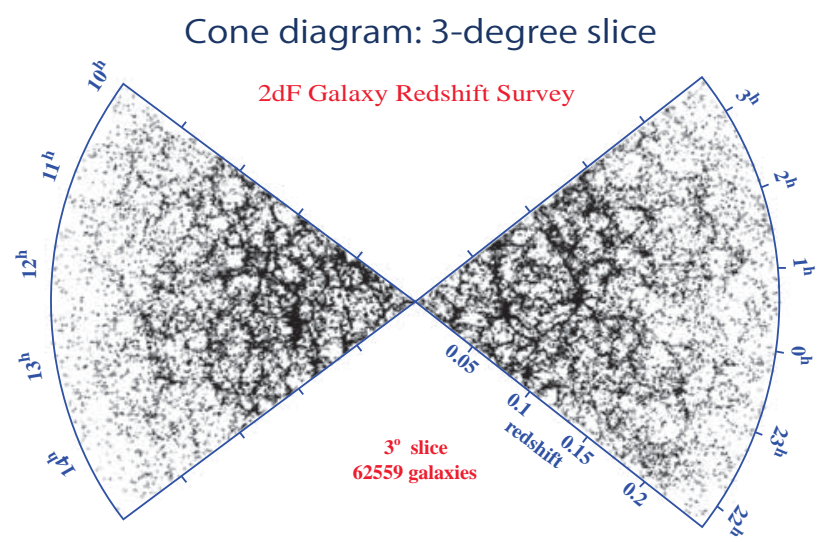

Fig. (23). Slice of $75^{\circ}-3^{\circ}$ in the $2 \mathrm{dFGRS}$. This plot contains 62559 galaxies and belongs to the 2dFGRS Image Gallery available at the web site: http://msowww.anu.edu.au/2dFGRS/. 


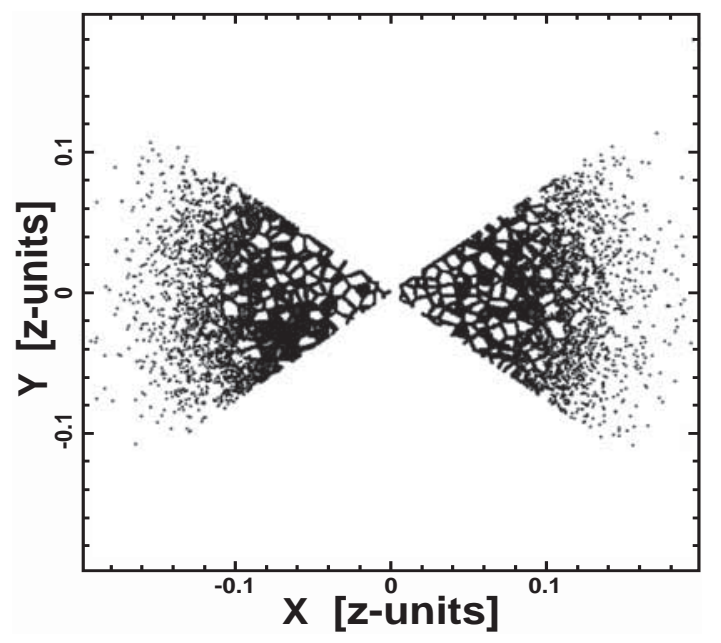

Fig. (24). Polar plot of the pixels belonging to a slice $75^{\circ}$ long and $3^{\circ}$ wide. This plot contains 62563 galaxies, the maximum in the frequencies of theoretical galaxies is at $\mathrm{z}=0.043$. In this plot $\mathrm{M}=$ 5.33 and $h=0.623$.

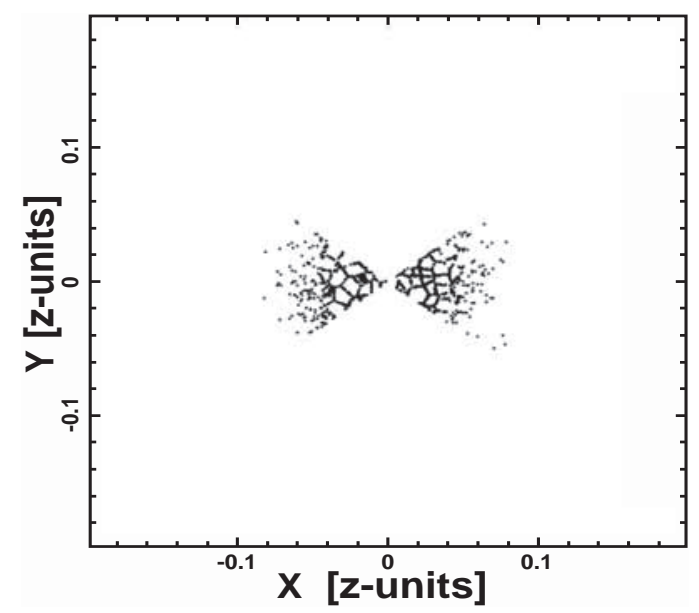

Fig. (25). Polar plot of the pixels belonging to a slice $75^{\circ}$ long and $3^{\circ}$ wide. Galaxies with magnitude $15.02<$ bJmag $<15.31$ or $46767<\frac{L_{\odot}}{M p c^{2}}<61063 \frac{L_{\odot}}{M p c^{2}}$. The maximum in the frequencies of theoretical galaxies is at $z=0.029, N_{S C}=2186$ and $N_{C}=62559$. In this plot $M_{\odot}=5.33$ and $h=0.623$.

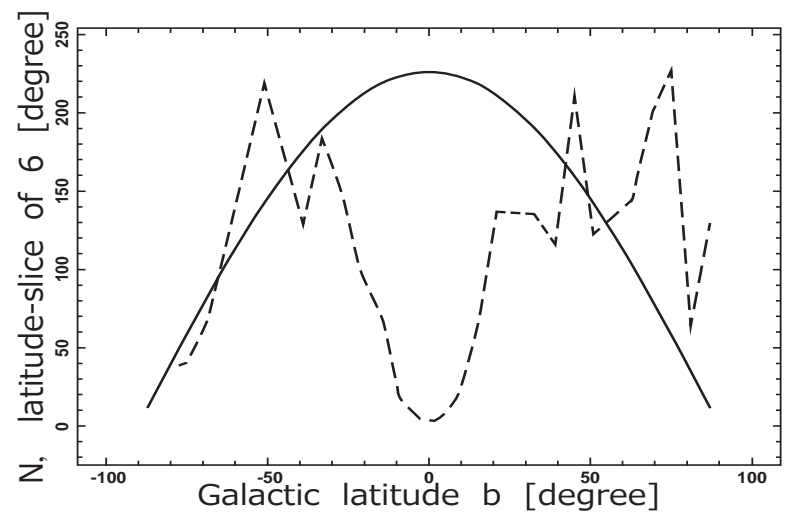

Fig. (26). The galaxies in the RC3 which have BT and redshift are organized in frequencies versus galactic latitude $\mathrm{b}$ (dashed line). The theoretical fit represents $\mathrm{Ni}$ (full line).

\subsection{The Third Reference Catalog of Bright Galaxies}

We now test the concept of an isotropic universe. This can be by done by plotting the number of galaxies comprised in a slice of $360^{\circ}$ in galactic longitude versus a variable number $\Delta b$ in galactic latitude, for example $6^{\circ}$. The number of galaxies in the RC3 versus galactic latitude is plotted in Fig. (26).

The solid angle $d \Omega$ in spherical coordinates $(\mathrm{r}, \theta, \phi)$ is

$d \Omega=\sin (\theta) d \theta d \varphi$

Table 13. Real and Simulated Data without the Zone of Avoidance in the RC3 Catalog

\begin{tabular}{|c|c|c|}
\hline & RC3 & No ZOA \\
\hline Elements & $\mathbf{3 3 1 6}$ & $\mathbf{4 3 2 6}$ \\
\hline \hline$Z_{\min }$ & $5.7 \times 10^{-7}$ & $8.9 \times 10^{-3}$ \\
\hline$Z_{\text {pos-max }}$ & $5.6 \times 10^{-3}$ & $8.9 \times 10^{-2}$ \\
\hline$Z_{\text {ave }}$ & $1.52 \times 10^{-2}$ & $7.96 \times 10^{-2}$ \\
\hline$Z_{\max }$ & $9.4 \times 10^{-2}$ & 0.14 \\
\hline
\end{tabular}

In a slice of $360^{\circ} \times \Delta b$ the amount of solid angle, $\Delta \Omega$, is

$\Delta \Omega=2 \pi\left(\left(\cos \left(90^{\circ}\right)-\cos (b+\Delta b)\right)-\left(\cos \left(90^{\circ}\right)-\cos (b)\right)\right)$ steradians

The approximate number of galaxies in each slice can be found through the following approximation. Firstly, we find the largest value of the frequencies of galaxies, $F_{i}$, versus $b$, $\max \left(F_{i}\right)$ where the index $i$ denotes a class in latitude. We therefore find the largest value of $\Delta \Omega_{i}, \max \left(\Delta \Omega_{i}\right)$. The introduction of the multiplicative factor $M$

$M=\frac{\max \left(F_{i}\right)}{\max \left(\Delta \Omega_{i}\right)}$

obtains the following theoretical evaluation of the number of galaxies $N_{i}$ as a function of the latitude,

$N_{i}=M \times \Delta \Omega_{i}$

This number, $N_{i}$, as a function of $b$ is plotted in Fig. (26).

The simulation of this overall sky survey can be done in the following way:

- The pixels belonging to the faces of irregular polyhedron are selected according to the distribution in $z$ of the galaxies in the RC3 catalog which have redshift and BT.

- A second operation selects the pixels according to the distribution in latitude in the RC3 catalog, see Fig. (27).

- In order to simulate a theoretical distribution of objects which represent the $\mathrm{RC} 3$ catalog without the Zone of Avoidance we made a series of $6^{\circ}$ slices in latitude in the RC3 catalog, selecting $N_{i}$ pixels in each slice, see Fig. (28). In order to ensure that the range in $z$ is correctly described Table 13 reports $z_{\min }, z_{\text {pos-max }}, z_{\text {ave }}$ and $z_{\max }$ which represent the minimum $z$, the position in $z$ of the maximum in the number of galaxies, and the maximum $z$ in the RC3 catalog or the simulated sample. 


\subsection{The CFA2 Catalog}

The results of the simulation can be represented by a slice similar to that observed (a strip of $6^{\circ}$ wide and about $130^{\circ}$ long), see Fig. (29).

A typical polar plot once the "scaling" algorithm is implemented, see [35], is reported in Fig. (30); it should be compared with the observations, see Fig. (31).

Fig. (32) reports both the CFA2 slice as well the simulated slice.

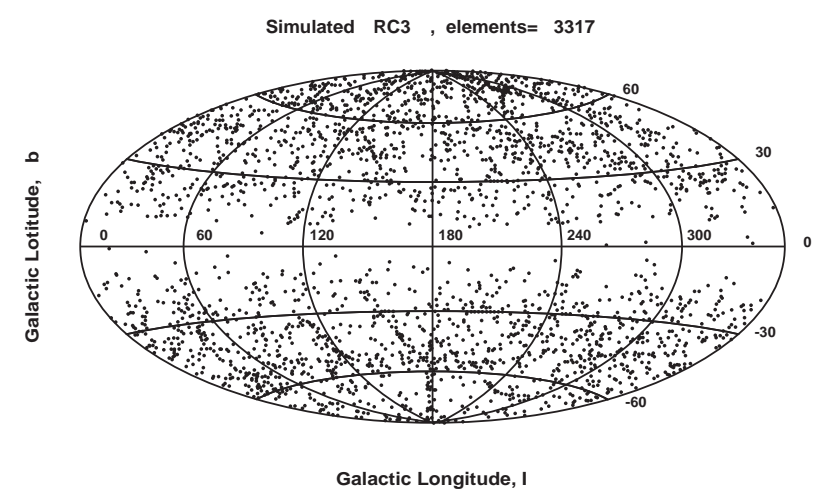

Fig. (27). Hammer-Aitoff projection of 3317 pixels belonging to a face of an irregular Voronoi Polyhedron. The Zone of Avoidance at the galactic plane follows Fig. (2). This plot simulates the RC3 galaxies which have BT and redshift. The galaxies are extracted according to the integral of the Schechter function in flux (formula (15) with parameters as in Table 2).

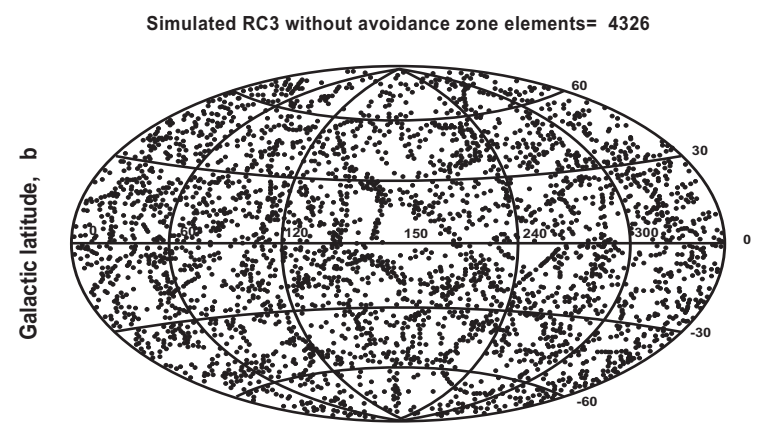

Galactic longitude, I

Fig. (28). Hammer-Aitoff projection of 4326 pixels belonging to a face of an irregular Voronoi Polyhedron. This plot simulates the RC3 galaxies which have BT and redshift but the Zone of Avoidance at the galactic plane is absent. The galaxies are extracted according to the integral of the Schechter function in flux (formula (15) with parameters as in Table 2)

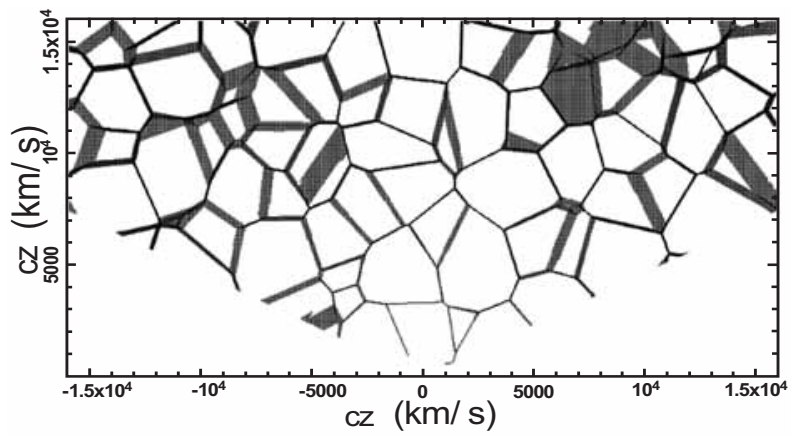

Fig. (29). Polar plot of the little cubes belonging to a slice 130 long and 6 wide.

\subsection{The Eridanus Supervoid}

A void can be defined as the empty space between filaments in a slice and the typical diameter has a range of [11-50] Mpc/h, see [90] and [91]. The probability, for example, of having a volume 3 times bigger than the average is $3.210^{-3}$ for PDF (55) when $d=3$ and $2.110^{-3}$ for PDF (47) when $d=2.75$. Particularly large voids are called supervoids and have a range of $[110-163] \mathrm{Mpc} / \mathrm{h}$.

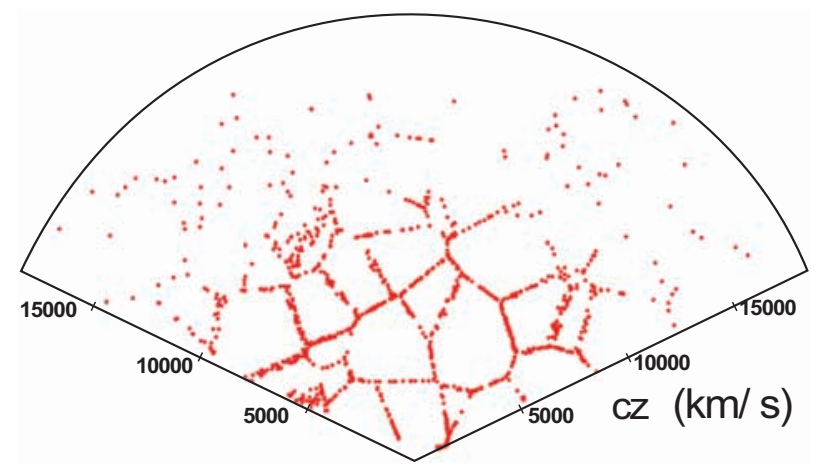

Fig. (30). Polar plot of the little cubes (red points) when the "scaling" algorithm is applied. Parameters as in Figure 29. This plot simulates the CFA2 slice.

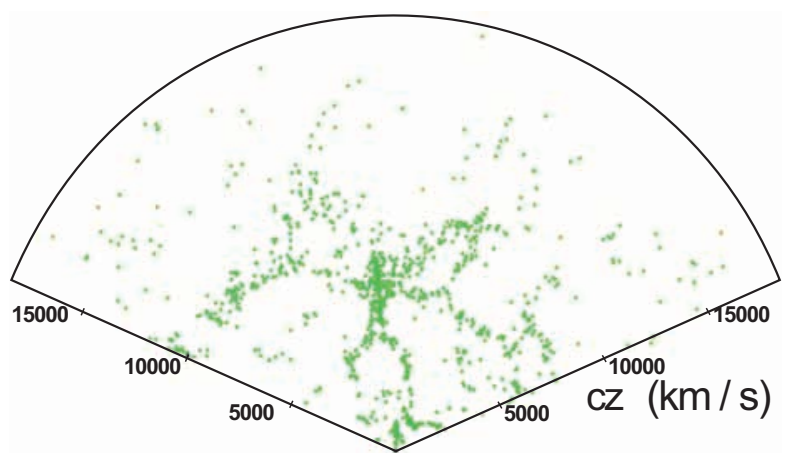

Fig. (31). Polar plot of the real galaxies (green points) belonging to the second CFA2 redshift catalog.

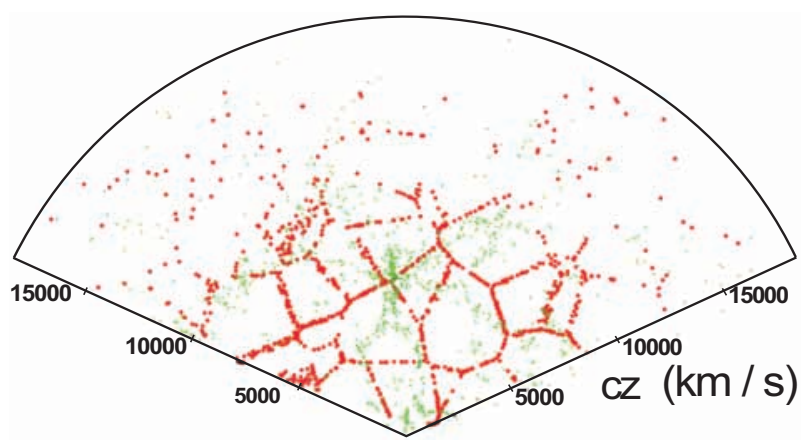

Fig. (32). Polar plot of the real galaxies (green points) belonging to the second CFA2 redshift catalog and the little cubes (red points).

Special attention should be paid to the Eridanus supervoid of $300 M p c$ in diameter. This super-void was detected by the Wilkinson Microwave Anisotropy Probe (WMAP), see [92-94], and was named Cold Spot. The WMAP measures the temperature fluctuations of the cosmic microwave background (CMB). Later on the astronomers 
confirmed the largest void due to the fact that the density of radio sources at $1.4 \mathrm{GHz}$ is anomalously low in the direction of the Cold Spot, see [95] and [96]. The standard statistics of the Voronoi normalized volume distribution in 3D covers the range [0.1-10]. In the case of a Eridanus super-void the normalized volume is $\approx \frac{300}{27}=1.3710^{3}$ and the connected probability of having such a super-void is $1.4710^{-18}$ when the Ferenc \& Neda function with $d=3$, formula (55), is used and $\approx 0$ when the Kiang function with $d=2.75$, formula (47) is used.

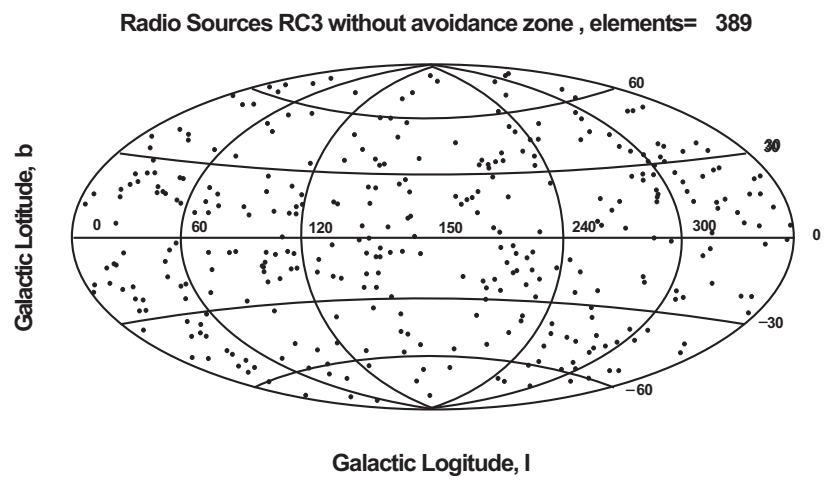

Fig. (33). Hammer-Aitoff projection of the SDSS-FIRST sources with complex radio morphology belonging to the RC3, prs $=0.09$. Other parameters as in Fig. (28).

Due to this low probability of having such a large normalized volume we mapped a possible spatial distribution of the SDSS-FIRST (the Faint Images of the Radio Sky at Twenty cm survey) sources with complex radio morphology from the theoretical distribution of galaxies belonging to the $\mathrm{RC} 3$. The fraction of galaxies belonging to the 2dFGRS detected as SDSS-FIRST sources with complex radio morphology is less than $10 \%$ according to Section 3.8 in [97]. We therefore introduced a probability, $p_{r s}$, that a galaxy is a radio source. The number of SDSS-FIRST sources $N_{r s}$ in the RC3 which are SDSS-FIRST sources with complex radio morphology is

$N_{r s}=p_{r s} * N_{g}$

where $N_{g}$ is the number of galaxies in the theoretical RC3.

From a visual inspection of Fig. (28) and Fig. (33) it is possible to conclude that the voids increase in size when radiogalaxies which are a subset of the galaxies are considered.

\section{THE CORRELATION FUNCTION FOR GALAXIES}

Galaxies have the tendency to be grouped in clusters and a typical measure is the computation of the two-points correlation function for galaxies, see $[98,6]$. The correlation function can be computed in two ways: a local analysis in the range [0-16] Mpc/h and an extended analysis in the range [0-200]Mpc/h.

\subsection{The Local Analysis}

A first way to describe the degree of clustering of galaxies is the two point correlation function $\xi_{G G}(r)$, usually presented in the form
$\xi_{G G}=\left(r r_{G}\right)^{-\gamma}$

where $\gamma_{G G}=1.8$ and $r_{G}=5.77 h^{-1} M p c$ (the correlation length) when the range $0.1 h^{-1} M p c<r<16 h^{-1} M p c$ is considered, see [99] where 118149 galaxies were analyzed.

In order to compute the correlation function, two volumes were compared: one containing the little cubes belonging to a face, the other containing a random distribution of points. From an analysis of the distances of pairs, the minimum and maximum were computed and $n_{D D}(r)$ was obtained, where $n_{D D}(r)$ is the number of pairs of galaxies with separation within the interval $[r-d r / 2, r+d r / 2]$. A similar procedure was applied to the random elements in the same volume with the same number of elements and $n_{R R}(r)$ is the number of pairs of the Poissonian Process. According to formula (16.4.6) in [100] the correlation function is:

$\xi_{G G}(r)=\frac{n_{D D}(r)}{n_{R R}(r)}-1$

To check whether $\xi_{G G}$ obeys a power law or not we used a simple linear regression test with the formula:

$\log \xi_{G G}=a+b \log r$

which allows us to compute $r_{G}=10^{-a / b}$ and $\gamma_{G G}=-\mathrm{b}$.

We now outline the method that allow us to compute the correlation function using the concept of thick faces, see [101]. A practical implementation is to consider a decreasing probability of having a galaxy in the direction perpendicular to the face. As an example we assume a probability, $p(x)$, of having a galaxy outside the face distributed as a Normal (Gaussian) distribution.

$p(x)=\frac{1}{\sigma(2 \pi)^{1 / 2}} \exp -\frac{x^{2}}{2 \sigma^{2}}$

where $x$ is the distance in $M p c$ from the face and $\sigma$ the standard deviation in $M p c$. Once the complex 3D behavior of the faces of the Voronoi Polyhedron is set up we can memorize such a probability on a $3 \mathrm{D}$ grid $P(i, j, k)$ which can be found in the following way

- In each lattice point $(i, j, k)$ we search for the nearest element belonging to a Voronoi face. The probability of having a galaxy is therefore computed according to formula (120).

- A number of galaxies, $N_{G}=n_{*} \times s i d e^{3}$ is then inserted in the box; here $n_{*}$ represents the density of galaxies.

Fig. (34) visualizes the edges belonging to the Voronoi diagrams and Fig. (35) represents a cut in the middle of the probability, $P(i, j, k)$, of having a galaxy to a given distance from a face.

A typical result of the simulation is reported in Fig. (36) where the center of the smaller box in which the correlation function is computed is the point belonging to a face nearest to the center of the big box. 


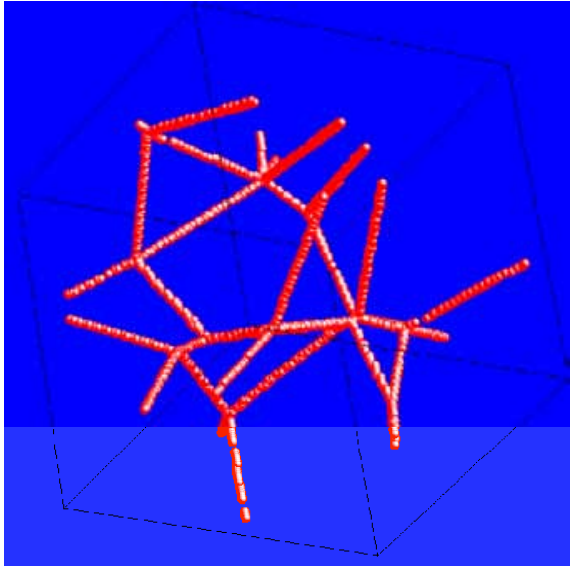

Fig. (34). 3D visualization of the edges of the Poissonian Voronoidiagram. The parameters are pixels $=60, \mathrm{Ns}=12$, side $=96.24$ $\mathrm{Mpc}, \mathrm{h}=0.623$ and amplify $=1.2$.

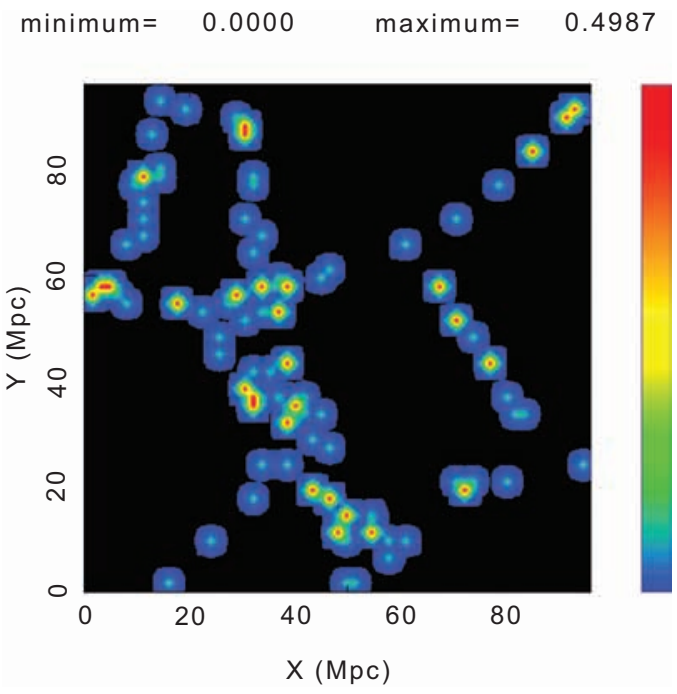

Fig. (35). Cut in the middle of the 3D grid $P(i, j, k)$ which represents a theoretical 2D map of the probability of having a galaxy. The Voronoi parameters are the same as in Fig. (34) and $\sigma=$ $0.8 \mathrm{Mpc}$. The $\mathrm{X}$ and $\mathrm{Y}$ units are in Mpc.

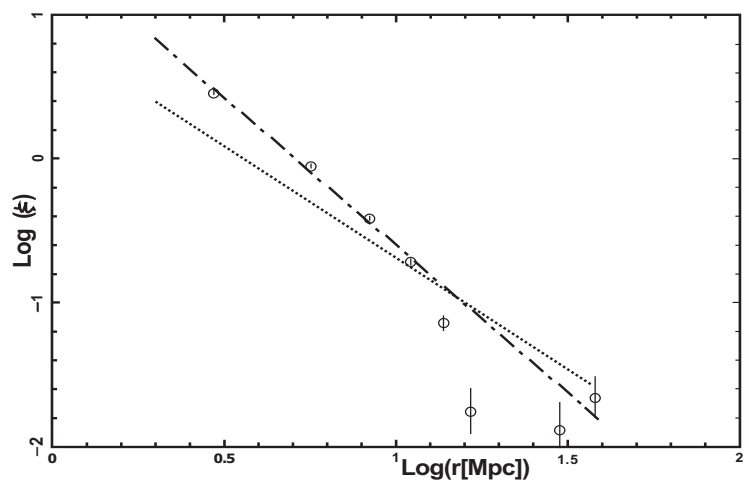

Fig. (36). The logarithm of the correlation function is visualized through points with their uncertainty (vertical bar), the asymptotic behavior of the correlation function $\xi_{G G}$ is reported as dash-dot-dash line; in our simulation $\gamma_{G G}=2.04$ and $r_{G}=5.08 \mathrm{Mpc}$. The standard value of the correlation function is reported as a dotted line; from the point of view of the observations in average $\gamma_{G G}=1.08$ and $r_{G}=5$ Mpc. Parameters of the simulation as in Fig. (34).
From an analysis of Fig. (36) we can deduce that the correlation function $\xi_{G G}$ of the simulation has a behavior similar to the standard one. Perhaps the value $r_{G}$ is a simple measure of the face's thickness, $\Delta R_{F}$. From this point of view on adopting a standard value of the expanding shell thickness, $\Delta R=\frac{R}{12}$ and assuming that the thickness of the shell is made by the superposition of two expanding shells the following is obtained

$\Delta R_{F} \approx \frac{R}{6} \approx \frac{\overline{D^{o b s}}}{h 12}=3.62 \mathrm{Mpc}$

where $h=0.623$ has been used. The correlation dimension $D_{2}$, see [1], is connected with the exponent $\gamma$ through the relation:

$D_{2}=3-\gamma$

Here there is the case in which the mass M(r) increases as $r^{1.2}$, in the middle of a one dimensional structure ( $M(r) \propto r)$ and a two dimensional sheet $\left(M(r) \propto r^{2}\right)$, see [100]. In this paragraph the dependence of the correlation function on the magnitude is not considered.

\subsection{The Extended Analysis}

A second definition of the correlation function takes account of the Landy-Szalay border correction, see [102],

$\xi_{L S}(s)=1+\frac{n_{D D}(s)}{n_{R R}(s)}-2 \frac{n_{D R}(s)}{n_{R R}(s)}$

where $n_{D D}(s), n_{D D}(s)$ and $n_{D R}(s)$ are the number of galaxy-galaxy, random-random and galaxy-random pairs having distance $s$, see [103]. A random catalog of galaxies in polar coordinates can built by generating a first random number $\propto z^{2}$ in the $z$-space and a second random angle in the interval $[0,75]$. A test of our code for the correlation function versus a more sophisticated code is reported in Fig. (37) for the $2 \mathrm{dFVL}$ volume limited (VL) sample, where the data available at the Web site http://www.uv.es/martinez/ have been processed.

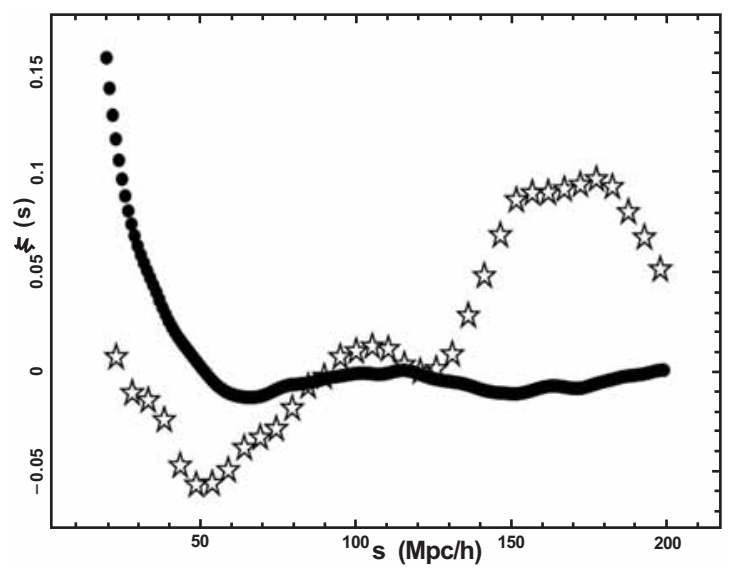

Fig. (37). Redshift-space correlation function for the 2dFGRS sample limited at $\mathrm{z}=0.12$ as given by our code (empty stars) and the 
results of [103] (full points) for 2dFVL. The covered range is [40 200] $\mathrm{Mpc} / \mathrm{h}$.

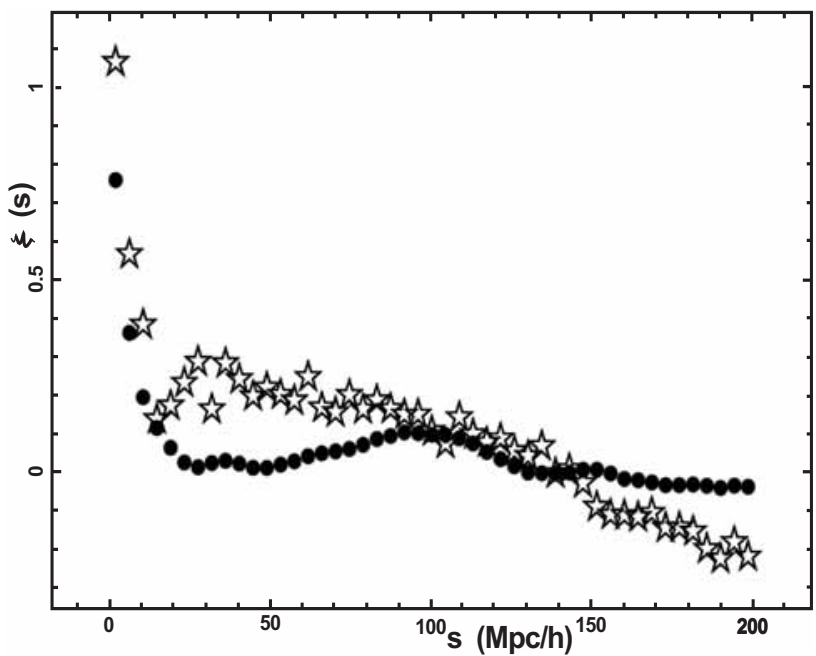

Fig. (38). Redshift-space correlation function for the 2dfGRS sample (empty stars) and the Voronoi sample (full points). The covered range is $[40-200] \mathrm{Mpc} / \mathrm{h}$.

The pair correlation function for the vertexes of the Poissonian Voronoi Polyhedron presents a typical damped oscillation, see Fig. $(\mathbf{5}, \mathbf{4}, \mathbf{1 1})$ in [104], Fig. (2) in [103] and Fig. (3) in [105]. Here conversely: (a) we first consider a set of objects belonging to the faces of the irregular Polyhedron; (b) we extract from the previous set a subset which follows the photometric law and then we compute the pair correlation function. The difference between our model and the model in [103] for 2dFVL can be due to the luminosity color segregation presents in $2 \mathrm{dFVL}$ but not in our Voronoi type model. A typical result is reported in Fig. (38) where it is possible to find the correlation function of 2 dfGRS with astronomical data as reported in Fig. (22) as well as the correlation function of the Voronoi network with simulated data as reported in Fig. (24).

A careful analysis of Fig. (38) allows us to conclude that the behavior of the correlation function is similar for the astronomical data as well as the simulated Voronoi-data. The oscillations after $100 \mathrm{Mpc}$ are classified as acoustic, [106].

\section{CONCLUSIONS}

\subsection{Photometric Maximum}

The observed number of galaxies in a given solid angle with a chosen flux/magnitude versus the redshift presents a maximum that is a function of the flux/magnitude. From a theoretical point of view, the photometric properties of the galaxies depend on the chosen law for the $L F$. The three $L F \mathrm{~s}$ here adopted predict a maximum in the theoretical number of galaxies as a function of the redshift once the apparent flux/magnitude is fixed, for the Schechter $L F$ see formula (13), for the $\mathcal{M}-L L F$ see formula (20) and for the generalized gamma $L F$ see formula (30).

The theoretical fit representing the number of galaxies as a function of the redshift can be compared with the real number of galaxies of the 2dFGRS which is theoryindependent. The superposition of theoretical and observed fit is acceptable, see Fig. (6). Particular attention should be paid to the Malmquist bias and to equation (37) that regulates the upper value of the redshift that defines the complete sample.

\section{3D VORONOI DIAGRAMS}

The intersection between a plane and the 3D Voronoi faces is well known as $V_{p}(2,3)$. The intersection between a

slice of a given opening angle, for example $3^{\circ}$, and the 3D Voronoi faces is less known and has been developed in Section 6.2. This intersection can be calibrated on the astronomical data once the number of Poissonian seeds is such that the largest observed void matches the largest Voronoi volume. Here the largest observed void is 2700 $\mathrm{Km} / \mathrm{sec}$ and in order to simulate, for example, the 2dFGRS, 137998 Poissonian seeds were inserted in a volume of $(131908 \mathrm{Km} / \mathrm{sec})^{3}$. The intersection between a sphere and the 3D Voronoi faces represents a new way to visualize the voids in the distribution of galaxies, see Section 6.2. In this spherical cut the intersection between a sphere and the 3D Voronoi faces is no longer represented by straight lines but by curved lines presenting in some cases a cusp behavior at the intersection, see Fig. (12). In line of principle the spatial distribution of galaxies at a given redshift should follow such curved lines.

\section{STATISTICS OF THE VOIDS}

The statistical properties of the voids between galaxies can be well described by the volume distribution of the Voronoi Polyhedra. Here two distributions of probability were carefully compared: the old Kiang function here parametrized as a function of the dimension $d$, see formula (44), and the new distribution of Ferenc \& Neda, see formula (55), which is a function of the selected dimension $d$. The analysis of the normalized areas of $V_{p}(2,3)$ is a subject of research rather than a well-established fact and we have fitted them with the Kiang function and the exponential distribution. The $\chi^{2}$ value indicates that the exponential distribution fits more closely the normalized area distribution of $V_{p}(2,3)$ than does the Kiang function, see Table 4. This fact follows from the comparison between the exponential and Kiang distributions of the radius, see Fig. (14). Therefore, the one parameter survival function of the radius of the exponential distribution for $V_{p}(2,3), S_{E R 23}$, as represented by (78), may model the voids between galaxies.

\section{SIMULATIONS OF THE CATALOGS OF GALAXIES}

By combining the photometric dependence in the number of galaxies as a function of the redshift with the intersection between a slice and the Voronoi faces, it is possible to simulate the astronomical catalogs such as the 2dFGRS, see Section 9.1. Other catalogs such as the RC3 which covers all the sky (except the Zone of Avoidance) can be simulated through a given number of spherical cuts, for example 25, with progressive increasing redshift. This simulation is visible in Fig. (27) in which the theoretical influence of the 
Zone of Avoidance has been inserted, and in Fig. (28) in which the theoretical RC3 without the Zone of Avoidance has been modeled. Fig. (33) reports the subset of the galaxies which are radiogalaxies.

\section{CORRELATION FUNCTION}

The standard behavior of the correlation function for galaxies in the short range $[0-10 \mathrm{Mpc} / \mathrm{h}]$ can be simulated once 12 Poissonian seeds are inserted in a box of volume $(96.24 \mathrm{Mpc} / \mathrm{h})^{3}$. In this case the model can be refined by introducing the concept of galaxies generated in a thick face belonging to the Voronoi Polyhedron. The behavior of the correlation function in the large range $[40-200 \mathrm{Mpc} / \mathrm{h}]$ of the Voronoi simulations of the $2 \mathrm{dFGRS}$ presents minimum variations from the processed astronomical data, see Fig. (38). We now extract a question from the conclusions of [103]. "Third, the minimum in the large-distance correlation functions of some samples demands explanation: is it really the signature of voids?" Our answer is "yes". The minimum in the large scale correlation function is due to the combined effect of the large empty space between galaxies (the voids) and to the photometric behavior of the number of galaxies as a function of the red-shift.

\section{STEREOLOGICAL APPROACH}

In this review analytical formulas have been provided which model the distributions of the lengths and areas of the planar sections of three-dimensional Poisson Voronoi diagrams: in particular, it has been shown that they are related to the Meier $G$ function. This finding is consistent with the analytical results presented in [107], where it is proved that nonlinear combinations of gamma variables, such as products or quotients, have distributions proportional, or closely related, to the Meijer $G$ distribution. The analytical distributions $F_{r}$ and $F_{A}$ been compared with results of numerical simulations.

\section{CONFLICT OF INTEREST}

The authors confirm that this article content has no conflicts of interest.

\section{ACKNOWLEDGEMENTS}

I thank the 2dF Galaxy Redshift Survey team for the use of Fig. (23), which is taken from the image gallery on the 2dFGRS website (see http://www2.aao.gov.au/2dFGRS) and the Stanford Encyclopedia of Philosophy for the use of Fig. (1).

\section{REFERENCES}

[1] JonesBJ, Mart'inezVJ, SaarE, TrimbleV. Scaling laws in the distribution of galaxies. Rev Mod Phys 2005; 76: 1211-66.

[2] Sparke LS, Gallagher JS. Galaxies in the universe: an introduction. Cambridge, UK: Cambridge University Press 2000.

[3] Mandelbrot B.On a decomposable model of a hierarchical universe-Derivation of galactic correlations on the celestial sphere. Acad Sci Paris C R Serie Sci Math 1975; 280: 1551-4.

[4] Soneira RM, Peebles PJE. Is there evidence for aspatially homogeneous population of feld galaxies? Astrophys J 1977; 211: 1-15.

[5] Soneira RM, Peebles PJE. A computer model universe-simulation of the nature of the galaxy distribution in the lick catalog. Astron J 1978; 83: 845-9.
[6] Peebles PJE. The large-scale structure of the universe. Princeton NJ: Princeton University Press 1980.

[7] Charlton JC, Schramm DN. Percolation of explosive galaxy formation. Astrophys J 1986; 310: 26-39.

[8] Zaninetti L, FerraroM. Transient phenomena and cluster formation. Astron Astrophys 1990; 239: 1-2.

[9] Klypin A, Shandarin SF. Percolation technique for galaxy clustering. Astrophys J 1993; 413: 48-58.

[10] Berlind AA, Frieman J, Weinberg DH, et al. Percolation galaxy groups and clusters in the sdss red shift survey: identifcation, catalogs, and the multiplicity function. Astrophys J 2006; 167: 1-25.

[11] Vogeley MS,Geller MJ, Huchra JP. Void statistics of the CfA red shift survey. Astrophys J 1991; 382: 44-54.

[12] Vogeley MS, Geller MJ, Park C, Huchra JP. Void sand constraints on nonlinear clustering of galaxies. Astron J 1994; 108: 745-58.

[13] Hoyle F, Vogeley MS. Voids in the point source catalogue survey and the updated zwicky catalog. Astrophys J 2002; 566: 641-51.

[14] Pan DC, Vogeley MS,Hoyle F,Choi YY,Park C. Cosmic voids in sloan digital sky survey data release7.ArXive-prints: 11034156.2011.

[15] Voronoi GF. Nouvelles applications des paramètres continus à la théorie des formes quadratiques. J Reine Angew Math 1907; 133: 97-178.

[16] Voronoi GF. Nouvelles applications des paramètres continus à la théorie de formes quadratiques. Z Reine Angew Math 1908; 134: 198-287.

[17] Descartes R. Principia Philosophiae. Amsterdam: LudovicusElzevirius 1644 .

[18] Aurenhammer F, Klein R. In: Sack J-R, Ed.Voronoidiagrams. Amsterdam: North-Holland 2000.

[19] Kiang T. Random Fragmentation in Two and Three Dimensions Z. Astrophys J 1966; 64: 433-39.

[20] IckeV, vande Weygaert R. Fragmenting the universe. Astron Astrophys 1987; 184: 16-32.

[21] vande Weygaert R, Icke V. Fragmenting the universe.II- Voronoi verticesas Abell clusters. Astron Astrophys 1989; 213: 1-2.

[22] Pierre M. Probes for the large-scale structure. Astron Astrophys 1990; 229: 7-16.

[23] Barrow JD, Coles P. The statistics of primordial density f uctuations. Mon Not R Astron Soc 1990; 244: 188-92.

[24] Coles P, Barrow JD. Microwave back ground constraints on the Voronoi model of large-scale structure. Mon Not R Astron Soc 1990; 244: 557-62.

[25] Coles P. Understanding recent observations of the large-scale stru of the universe. Nature 1990; 346: 446.

[26] vande Weygaert R. Quasi-periodicity in deep red shift surveys. Mon Not R Asron Soc 1991; 249: 159-63.

[27] vande Weygaert R. Voids and the large scale structure of the universe. PhD thesis, Leiden: University of Leiden 1991.

[28] Zaninetti L. Dynamical Voronoite ssellation.III-The distribution of galaxies. Astron Astrophys 1991; 246: 291-300.

[29] Ikeuchi S, Turner EL. Quasi-periodic structures in the large-scale galaxy distribution and three-dimensional Voronoites sellation. Mon Not R Astron Soc 1991; 250: 519-22.

[30] Subba Rao MU, SzalayAS. Statistics of pencil beamsin Voronoifoams. Astrophys J 1992; 391: 483-93.

[31] van de Weygaert R. Fragmenting the Universe. 3: The constructions and statistics of 3-D Voronoi tessellations. Astron Astrophys 1994; 283: 361-406.

[32] Goldwirth DS, da Costa LN, van de Weygaert R. The two-point correlation function and the size of voids. Mon Not R Astron Soc 1995; 275: 1185-95.

[33] van de Weygaert R. Froth across the universe dynamics and stochastic geometry of the cosmic foam. arXiv: astro-ph/0206427. 2002.

[34] van de Weygaert R. In: statistics of galaxy clustering - commentary. Stat Challeng Astron 2000; pp. 156-86.

[35] Zaninetti L. On the large-scale structure of the Universe as given by the Voronoi Diagrams. Chin J Astron Astrophys 2006; 6: 38795.

[36] Ebeling H, Wiedenmann G. Detecting structure in two dimensions combining Voronoi tessellation and percolation. Phys Rev E 1993; 47: 704-10.

[37] Bernardeau F, van de Weygaert R. A new method for accurate estimation of velocity field statistics. Mon Not R Astron Soc 1996; 279: 693-701. 
[38] Schaap WE, van de Weygaert R. Continuous fields and discrete samples: reconstruction through Delaunay tessellations. Astron Astrophys 2000; 363: L29-L32.

[39] Marinoni C, Davis M, Newman JA, Coil AL. Three-dimensional identification and reconstruction of galaxy systems within fluxlimited redshift surveys. Astrophys J 2002; 580: 122-43.

[40] Melnyk OV, Elyiv AA, Vavilova IB. The structure of the local supercluster of galaxies detected by three-dimensional Voronoi's tessellation method. Kinemat Fizika Nebesnykh Tel 2006; 22: 28396.

[41] van de Weygaert R, Schaap W. The cosmic web: geometric analysis. In: Martinez VJ, Saar E, Gonzales EM, Pons-Borderia MJ, Eds. Berlin: Springer Verlag 2009; pp. 291-311.

[42] Elyiv A, Melnyk O, Vavilova I. High-order 3D Voronoi tessellation for identifying isolated galaxies, pairs and triplets. Mon Not R Astron Soc 2009; 394: 1409-18.

[43] Aarseth SJ. Computer simulations of galaxy clustering. In: The large scale structure of the Photometric Effects and Voronoidiagrams as a mixed model for the spatial distribution of galaxies 45 universe.1978; p. 189-96.

[44] Turner EL, Aarseth SJ, Blanchard NT, Mathieu RD, Gott JR III. Nbody simulations of galaxy clustering. II - Groups of galaxies. Astrophys J 1979; 228: 684-95.

[45] Gott JR III, Turner EL, Aarseth SJ. N-body simulations of galaxy clustering. III - The covariance function. Astrophys J 1979; 234: 13-26.

[46] Evrard AE, MacFarland TJ, Couchman HMP, et al. Galaxy clusters in hubble volume simulations: cosmological constraints from sky survey populations. Astrophys J 2002; 573: 7-36.

[47] Peebles PJE. A model for continuous clustering in the large-scale distribution of matter. Astrophys Space Sci 1974; 31: 403-10.

[48] Peebles PJE. The gravitational-instability picture and the nature of the distribution of galaxies. Astrophys J 1974; 189: 51-9.

[49] Gott JR III, Rees MJ. A theory of galaxy formation and clustering. Astron Astrophys 1975; 45: 365-76.

[50] Zel'dovich YB. Gravitational instability: an approximate theory for large density perturbations. Astron Astrophys 1970; 5: 84-9.

[51] Shandarin SF, Zel'dovich YB. The large-scale structure of the universe: Turbulence, intermittency, structures in a self-gravitating medium. Rev Mod Phys 1989; 61: 185-220.

[52] Sahni V, Coles P. Approximation methods for non-linear gravitational clustering. Phys Rep 1995; 262: 1-135.

[53] Neyman J, Scott EL. A theory of the spatial distribution of galaxies. Astrophys J 1952; 116: 144-54.

[54] Scherrer RJ, Bertschinger E. Statistics of primordial density perturbations from discrete seed masses. Astrophys J 1991; 381: 349-60.

[55] Cooray A, Sheth R. Halo models of large scale structure. Phys Rep 2002; 372: 1-129.

[56] Hubble E. A relation between distance and radial velocity among extra-galactic nebulae. Proc Natl Acad Sci USA 1929; 15: 168-73.

[57] Sandage A, Tammann GA, Saha A, Reindl B, Macchetto FD, Panagia N. The hubble constant: a summary of the hubble space telescope program for the luminosity calibration of type Ia supernovae by means of cepheids. Astrophys J 2006; 653: 843-60.

[58] Mattig W. Uber den Zusammenhang zwischen Rotverschiebung und scheinbarer Helligkeit. Astron Nachri 1958; 284: 109-19.

[59] Lang KR. Astrophysical formulae $3^{\text {rd }}$ ed. New York: Springer 1999.

[60] Schechter P. An analytic expression for the luminosity functions for galaxies. Astrophys J 1976; 203: 297-306.

[61] Padmanabhan T. Cosmology and astrophysics through problems. Cambridge: Cambridge University Press 1996.

[62] Padmanabhan P. Theoretical astrophysics. Vol. III: galaxies and cosmology. Cambridge, MA: Cambridge University Press 2002.

[63] Zaninetti L. A new luminosity function for galaxies as given by the mass-luminosity relationship. Astron J 2008; 135: 1264-75.

[64] Evans M, Hastings N, Peacock B. Statistical distribution. $3^{\text {rd }}$ ed. New York: John Wiley \& Sons Inc 2000.

[65] Zaninetti L. The luminosity function of galaxies as modelled by the generalized gamma distribution. Acta Phys Polon B 2010; 41(4): 729-51.

[66] de Vaucouleurs G, de Vaucouleurs A, Corwin HG Jr, Buta RJ, Paturel G, Fouque P. Third reference catalogue of bright galaxies. New York,NY: Springer-Verlag 1991.

[67] Jarrett T. Large scale structure in the local universe - the 2MASS galaxy catalog. PASA 2004; 21: 396-403.
[68] Crook AC, Huchra JP, Martimbeau N, Masters KL, Jarrett T, Macri LM. Groups of galaxies in the two micron all sky redshift survey. Astrophys J 2007; 655: 790-813.

[69] Geller MJ, Huchra JP. Mapping the universe. Science 1989; 246: 897-903.

[70] Malmquist KG. A study of the stars of spectral type A. Lund Medd Ser II 1920; $22: 1-10$.

[71] Malmquist KG. On some relations in stellar statistics. Lund Medd Ser I 1922; 100: 1-10.

[72] Behr A. Zur Entfernungsskala der extragalaktischen Nebel. Astron Nachri 1951; 279: 97-107.

[73] Zaninetti L. A geometrical model for the catalogs of galaxies . Rev Mexicana Astron Astro 2010; 46: 115-34.

[74] Neyrinck MC, Gnedin NY, Hamilton AJS. VOBOZ: an almostparameter-free halo-finding algorithm. Mon Not R Astron Soc 2005; 356: 1222-32.

[75] Press WH, Teukolsky SA, Vetterling WT, Flannery BP. Numerical Recipes in FORTRAN. The Art of Scientific Computing. Cambridge: Cambridge University Press 1992.

[76] Hinde AL, Miles RE. Monte Carlo estimates of the distributions of the random polygons of the Voronoi tessellation with respect to a Poisson process . J Stat Comput Simul 1980; 10: 205-23.

[77] Ferenc JS, N'eda Z. On the size distribution of Poisson Voronoi cells. Phys A 2007; 385: 518-26.

[78] Tanemura M. Statistical Distributions of Poisson Voronoi Cells in Two and Three Dimensions. Forma 2003; 18: 221-47.

[79] Zaninetti L. Practical statistics for the voids between galaxies. Serbian Astron J 2010; 181: 19-29.

[80] Møller J. Random tessellations in d. Adv Appl Probab 1989; 21(1): 37-73.

[81] Møller J. Lectures on random Voronoi tessellations. Lecture Notes in Statistics (Springer).87. New York, NY: Springer- Verlag 1994; vol. 134: p. DM 49.00.

[82] Chiu SN, Weygaert RVD, Stoyan D. The sectional poisson voronoi tessellation is not a voronoi tessellation. Adv Appl Prob 1996; 28(2): 356-76.

[83] Blower JD, Keating JP, Mader HM, Phillips JC. The evolution of bubble size distributions in volcanic eruptions. J Volcanol Geotherm Res 2002; 120(1-2): 1-23.

[84] Ferraro M, Zaninetti L. Statistics of cross sections of Voronoi tessellations. Phys Rev E 2011; 84(4): 041107.

[85] Meijer CS. Uber Whittakersche bzw. Besselsche Funktionen und deren Produkte. Nieuw Arch Wiskd 1936; 18: 10-39.

[86] Meijer CS. Multiplikationstheoreme fur die Funktion Gm,n p,q (z). Proc Akad Wet Amsterdam 1941; 44: 1062-70.

[87] Olver FWJe, Lozier DWe, Boisvert RFe, Clark CWe. NIST handbook of mathematical functions. Cambridge: Cambridge University Press 2010.

[88] Zaninetti L. New analytical results for poissonian and nonpoissonian statistics of cosmic voids. Rev Mex Astron Astrofis 2012; 48: 209-22.

[89] Brandt S, Gowan G. Data analysis: statistical and computational methods for scientists and engineers. New-York: Springer \& Verlag 1998.

[90] Einasto M, Einasto J, Tago E, Dalton GB, Andernach H. The structure of the universe traced by rich clusters of galaxies. Mon Not R Astron Soc 1994; 269: 301-22.

[91] Lindner U, Einasto J, Einasto M, Freudling W, Fricke K, Tago E. The structure of supervoids. I. Void hierarchy in the Northern Local Supervoid. Astron Astrophys 1995; 301: 329-47.

[92] Vielva P, Mart'inez-Gonz'alez E, Barreiro RB, Sanz JL, Cay’on L. Detection of non-gaussianity in the wilkinson microwave anisotropy probe first-year data using spherical wavelets. Astrophys J 2004; 609: 22-34.

[93] Cruz M, Mart'inez-Gonz'alez E, Vielva P, Cay’on L. Detection of a non-Gaussian spot in WMAP. Mon Not R Astron Soc 2005; 356 : 29-40.

[94] Vielva P. A comprehensive overview of the cold spot. Adv Astron 2010; 2010: 1-20.

[95] Rudnick L, Brown S, Williams LR. Extragalactic radio sources and the WMAP cold spot. Astrophys J 2007; 671: 40-4.

[96] McEwen JD, Wiaux Y, Hobson MP, Vandergheynst P, Lasenby AN. Probing dark energy with steerable wavelets through correlation of WMAP and NVSS local morphological measures. Mon Not R Astron Soc 2008; 384: 1289-300. 
[97] Ivezíč Z, Menou K, Knapp GR, Strauss MA, Lupton RH. Optical and radio properties of extragalactic sources observed by the FIRST survey and the sloan digital sky survey. Astron J 2002; 124: 2364-400.

[98] Peebles PJE. Principles of physical cosmology. Princeton, N.J: Princeton University Press 1993.

[99] Zehavi I, Weinberg DH, Zheng Z, Berlind AA, Frieman JA, Scoccimarro R. On departures from a power law in the galaxy correlation function. Astrophys J 2004; 608: 16-24.

[100] Coles P, Lucchin F. Cosmology: the origin and evolution of cosmic structure. $2^{\text {nd }}$ ed. Chichester, England: John Wiley \& Son 2002.

[101] Zaninetti L. Dynamical voronoi tessellation. V. thickness and incompleteness. Astro Astrophys 1995; 109: 71-7.

[102] Szalay AS, Broadhurst TJ, Ellman N, Koo DC, Ellis RS. Redshift survey with multiple pencil beams at the galactic poles. Proc Natl Acad Sci USA 1993; 90: 4853-8.
[103] Martínez VJ, Arnalte-Mur P, Saar E, et al. reliability of the detection of the baryon acoustic peak. Astrophys J 2009; 696: L93-97.

[104] Okabe A, Boots B, Sugihara K. Spatial tessellations: concepts and applications of voronoi photometric effects and voronoi-diagrams as a mixed model for the spatial distribution of galaxies 47 diagrams. Chichester, New York: Wiley 1992.

[105] Heinrich L, Muche L. Second-order properties of the point process of nodes in a stationary Voronoi tessellation. Math Nachr 2008; 281(3): 350-75.

[106] Eisenstein DJ, Zehavi I, Hogg DW, Scoccimarro R, Blanton MR, Nichol RCea. Detection of the baryon acoustic peak in the largescale correlation function of SDSS luminous red galaxies. Astrophys J 2005; 633: 560-74.

[107] Springer MD, Thompson WE. The distribution of products of beta gamma and Gaussian random variables. SIAM J Appl Math 1970; 18: 721-37.

(C) L. Zaninetti; Licensee Bentham Open.

This is an open access article licensed under the terms of the Creative Commons Attribution Non-Commercial License (http://creativecommons.org/licenses/by-nc/3.0/) which permits unrestricted, non-commercial use, distribution and reproduction in any medium, provided the work is properly cited. 